Comment. Math. Helv. 75 (2000) 368-409

$0010-2571 / 00 / 030368-42 \$ 1.50+0.20 / 0$
(C) 2000 Birkhäuser Verlag, Basel

Commentarii Mathematici Helvetici

\title{
On a problem of Nazarova and Roiter
}

\author{
Bangming Deng
}

\begin{abstract}
In the present paper we introduce the notion of representations of a bush which is a generalization of matrix problems (self-reproducing systems) introduced by Nazarova and Roiter. We show that the problem of classifying representations of clannish algebras come down to such generalized matrix problems. Based on the classification of Crawley-Boevey, we provide a description of indecomposable representations of bushes over any field. The proof is based on a categorical formulation of the matrix reduction of Nazarova and Roiter.
\end{abstract}

Mathematics Subject Classification (2000). 16G10, 16G20, 18A25.

Keywords. Rods, bushes, clannish algebras, self-reproducing systems.

\section{Introduction}

In the present article, we consider a generalization of matrix problems (selfreproducing systems) introduced by Nazarova and Roiter [8]. Their motivation was to solve a problem posed by Gelfand [6]: classify the indecomposable representations of the quiver

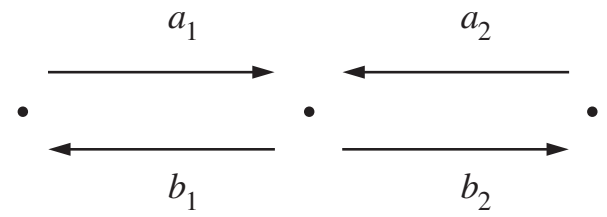

subjected to the relation $a_{1} b_{1}=a_{2} b_{2}$.

In [2] Crawley-Boevey reconsiders the problem and introduces a new class of matrix problems called "clans". The approach used in [2] is the functorial filtration method. It seems to us that both the notion of a clan and the functorial method are not well adapted to the problem treated by Crawley-Boevey.

Supported partially by the National Natural Science Foundation of China (Grant No.19971009) 
Our aim here is to replace clans by a generalization of self-reproducing systems and to use the method presented in [8] instead of the functorial one. Our method also works for fields of cardinality 2, a case which Crawley-Boevey was unable to handle with his method. Our classification however is based on that of CrawleyBoevey. For the proof of our classification theorem we use a categorical formulation of the matrix reduction of Nazarova and Roiter (see [3]).

After the completion of a preliminary version of the present paper, Prof. Sergejchuk pointed out to me that the matrix problems considered here have been studied by Bondarenko [1].

Throughout the paper, $k$ denotes an arbitrary field.

The terminology used throughout the paper is taken from [5].

\section{Tangles and Bushes}

1.1. Let $\mathcal{A}$ be an aggregate over a field $k$ with spectroid $\mathcal{S}$. A tangle over $\mathcal{A}$ is a pair $\left(M^{-}, M^{+}\right)$formed by sequences $M^{-}=\left(M_{1}^{-}, \cdots, M_{n}^{-}\right)$and $M^{+}=$ $\left(M_{1}^{+}, \cdots, M_{n}^{+}\right)$of pointwise finite left $\mathcal{A}$-modules. Given such a tangle, we denote by $\operatorname{rep}\left(M^{-}, M^{+}\right)$the aggregate whose objects are the representations of $\left(M^{-}, M^{+}\right)$, i.e. the sequences $\left(X ; f_{1}, \cdots, f_{n}\right)$ where $X \in \mathcal{A}$ and $f_{i} \in \operatorname{Hom}_{k}\left(M_{i}^{-}(X)\right.$, $\left.M_{i}^{+}(X)\right), i=1, \cdots, n$. A morphism from $\left(X ; f_{1}, \cdots, f_{n}\right)$ to $\left(X^{\prime} ; f_{1}^{\prime}, \cdots, f_{n}^{\prime}\right)$ is given by a morphism $\mu \in \mathcal{A}\left(X, X^{\prime}\right)$ such that $f_{i}^{\prime} M_{i}^{-}(\mu)=M_{i}^{+}(\mu) f_{i}$ for $i=$ $1, \cdots, n$.

1.2. Our aim is to classify the indecomposables of $\operatorname{rep}\left(M^{-}, M^{+}\right)$for particular tangles $\left(M^{-}, M^{+}\right)$which we describe now.

By definition, a rod is a finite ordered set $R$ such that each $x \in R$ admits at most one $y \in R$ satisfying $y \rtimes x$ (i.e. incomparable with $x$ ).

Examples. The ordered sets with the following Hasse-quivers are rods:
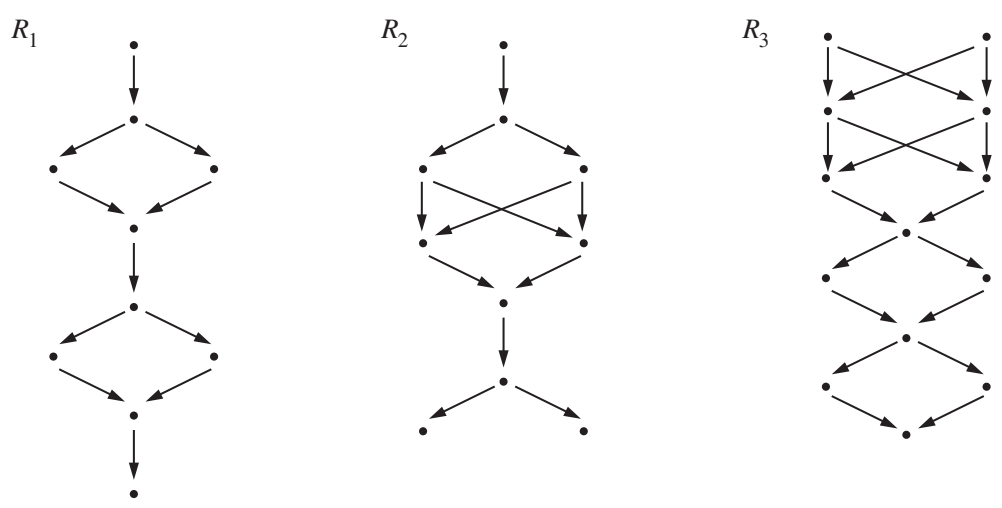
A tangle $\left(M^{-}, M^{+}\right)$is called rodded if the following conditions are satisfied: (R1) For each $i$, the lattices of submodules of $M_{i}^{-}$and $M_{i}^{+}$are rods;

$(\mathrm{R} 2)$ For each $s \in \mathcal{S}$,

$$
\sum_{i=1}^{n}\left(\operatorname{dim}_{k} M_{i}^{-}(s)+\operatorname{dim}_{k} M_{i}^{+}(s)\right) \leq 2
$$

(R3) If the submodules of $M_{i}^{\varepsilon}$ generated by elements $a \in M_{i}^{\varepsilon}(s)$ and $b \in M_{i}^{\varepsilon}(t)$ are incomparable for some $i$, some $s, t \in \mathcal{S}$ and some $\varepsilon \in\{-,+\}$, then

$$
\sum_{j, \eta} \operatorname{dim}_{k} M_{j}^{\eta}(s)=1=\sum_{j, \eta} \operatorname{dim}_{k} M_{j}^{\eta}(t) ;
$$

(R4) For any $s, t \in \mathcal{S}$, the canonical map

$$
\theta(s, t): \mathcal{R}_{\mathcal{S}}(s, t) \longrightarrow \prod_{i=1}^{n} \mathcal{R}_{i}^{-}(s, t) \times \mathcal{R}_{i}^{+}(s, t)
$$

is surjective.

Here $\mathcal{R}_{\mathcal{S}}$ denotes the radical of $\mathcal{S}$ and $\mathcal{R}_{i}^{\varepsilon}(s, t)$ the set of all $f \in \operatorname{Hom}_{k}\left(M_{i}^{\varepsilon}(s)\right.$, $\left.M_{i}^{\varepsilon}(t)\right)$ satisfying $f(N(s)) \subset \mathcal{R} N(t)$ for each submodule $N$ of $M_{i}^{\varepsilon}$.

1.3. Given a tangle $\left(M^{-}, M^{+}\right)$over $\mathcal{A}$, we denote by $\mathcal{I}$ the intersection of the annihilators of all $M_{i}^{-}$and $M_{i}^{+}$. The tangle is called faithful if $\mathcal{I}=0$. In the case of a faithful rodded tangle, the maps $\theta(s, t)$ are bijective. Our purpose is to give a concrete construction of faithful rodded tangles.

Let $S$ be a pair formed by two sequences of disjoint rods $S^{-}=\left(S_{1}^{-}, \cdots, S_{n}^{-}\right)$ and $S^{+}=\left(S_{1}^{+}, \cdots, S_{n}^{+}\right)$. We then equip the union $|S|=\cup_{i=1}^{n}\left(S_{i}^{-} \cup S_{i}^{+}\right)$with the smallest order relation containing the order relations of the rods $S_{i}^{-}$and $S_{i}^{+}$. If there is no risk of confusion, we simply write $S$ instead of $|S|$. By $k S$ we denote the spectroid whose objects are the elements of $S$, whose morphism-spaces $k S(x, y)$ are one-dimensional with basis $(y \mid x)$ if $y \geq x$, or else are 0 . The composition is such that $(z \mid y) \circ(y \mid x)=(z \mid x)$ [5]. Each interval $I$ of $S$ gives rise to a module $k_{I}$ over $k S$ such that $k_{I}(x)=0$ if $x \notin I$ and $k_{I}(y)=k, k_{I}(z \mid y)=\mathbb{1}_{k}$ if $y, z \in I$ and $y \leq z[5]$. We set $L_{i}^{-}=k_{I}$ if $I=S_{i}^{-}$and $L_{i}^{+}=k_{I}$ if $I=S_{i}^{+}$.

Let further $\sim$ be an equivalence relation on $S$ such that:

(E1) Each equivalence class contains at most two elements;

(E2) In case $x, y \in S_{i}^{\varepsilon}$ and $x \gtrless y$, the equivalence class of $x$ consists of $x$ only.

The $S$ together with the equivalence relation is called a bush.

Let $\mathcal{S}$ denote the spectroid whose objects are the equivalence classes of $S$, whose spaces of radical morphisms are $\mathcal{R}_{\mathcal{S}}(a, b)=\oplus_{x \in a, y \in b, y>x} k(y \mid x)$, whose composition is such that $\left(z \mid y^{\prime}\right) \circ(y \mid x)$ is $(z \mid x)$ if $y^{\prime}=y$ and 0 otherwise. Let further $\mathcal{A}:=$ $\oplus \mathcal{S}$ denote the additive hull of $\mathcal{S}$, whose objects are sequences $\left(X_{1}, \cdots, X_{l}\right)$ of 
objects of $\mathcal{S}$, whose morphisms $\left(X_{1}, \cdots, X_{l}\right) \rightarrow\left(Y_{1}, \cdots, Y_{m}\right)$ are identified with the "matrices" $\mu=\left[\mu_{j i}\right] \in \oplus_{i, j} \mathcal{S}\left(X_{i}, Y_{j}\right)$. The composition of morphisms obeys the rules of matrix multiplication. Then each module $L$ over $k S$ provides a module $\tilde{L}$ over $\mathcal{A}$ such that $\tilde{L}(a)=\oplus_{x \in a} L(x)$ for each $a \in \mathcal{S}$; the action of $\tilde{L}(y \mid t)$ on $m \in L(x) \subset \tilde{L}(a)$ coincides with that of $L(y \mid x)$ if $x=t$ or else is 0 . In case $L=L_{i}^{-}$ (resp. $\left.L_{i}^{+}\right)$, we set $\tilde{L}=M_{i}^{-}\left(\right.$resp. $\left.M_{i}^{+}\right)$, thus obtaining a tangle $\left(M^{-}, M^{+}\right)$over $\mathcal{A}$. This tangle is faithful and rodded.

In the sequel, the representations of $\left(M^{-}, M^{+}\right)$will be simply called representations of $S$.

Proposition. For each faithful rodded tangle $\left(N^{-}, N^{+}\right)$over an aggregate $\mathcal{B}$ with spectroid $\mathcal{T}$, there is a bush $S$ as above and an equivalence $\Phi: \mathcal{A} \rightarrow \mathcal{B}$ such that $N_{i}^{\varepsilon} \Phi \cong M_{i}^{\varepsilon}$ for all $i \in\{1, \cdots, n\}$ and $\varepsilon \in\{-,+\}$.

Proof. Let the points of $S_{i}^{\varepsilon}$ be given by the submodules $X$ of $N_{i}^{\varepsilon}$ with simple top $X / \mathcal{R} X, i \in\{1, \cdots, n\}, \varepsilon \in\{-,+\}$. We equip $S_{i}^{\varepsilon}$ with an order relation such that $X \leq Y$ is equivalent to $X \supseteq Y$. By (R1), $S_{i}^{\varepsilon}$ is a rod.

Set $S=\left(S_{1}^{-}, \cdots, S_{n}^{-} ; S_{1}^{+}, \cdots, S_{n}^{+}\right)$and equip $S$ with an equivalence relation such that $X \sim Y \Longleftrightarrow X / \mathcal{R} X \cong Y / \mathcal{R} Y$. By (R2) and (R3), this relation satisfies (E1) and (E2), i.e. $S$ is a bush.

For each $X \in \mathcal{S}$ (the spectroid associated with the bush $S$ ), we denote by $t_{X} \in \mathcal{T}$ the point supporting $X / \mathcal{R} X$, and we choose a generator $e_{X} \in N_{i}^{\varepsilon}\left(t_{X}\right)$ of $X$. Then $N_{i}^{\varepsilon}\left(t_{X}\right)=\oplus_{X^{\prime} \sim X, X^{\prime} \in S_{i}^{\varepsilon}} k e_{X^{\prime}}$.

The map $X \longmapsto t_{X}$ gives rise to a functor $\phi: \mathcal{S} \rightarrow \mathcal{T}$ such that $\phi(Y \mid X)=$ $\theta\left(t_{X}, t_{Y}\right)^{-1}(f)$, where $f \in \operatorname{Hom}_{k}\left(N_{i}^{\varepsilon}\left(t_{X}\right), N_{i}^{\varepsilon}\left(t_{Y}\right)\right)$ maps $e_{X}$ to $e_{Y}$ and annihilates $e_{X^{\prime}}$ whenever $X^{\prime} \neq X$. The functor $\phi$ is an isomorphism and induces an equivalence $\Phi: \mathcal{A} \rightarrow \mathcal{B}$. The $k$-linear maps

$$
\begin{gathered}
\mu_{i}^{\varepsilon}(X): N_{i}^{\varepsilon} \Phi(X)=\bigoplus_{X^{\prime} \sim X, X^{\prime} \in S_{i}^{\varepsilon}} k e_{X^{\prime}} \longrightarrow \bigoplus_{X^{\prime} \sim X, X^{\prime} \in S_{i}^{\varepsilon}} k X^{\prime}=M_{i}^{\varepsilon}(X) \\
\sum_{X^{\prime}} \lambda_{X^{\prime}} e_{X^{\prime}} \longmapsto \sum_{X^{\prime}} \lambda_{X^{\prime}} X^{\prime}
\end{gathered}
$$

define an isomorphism between $N_{i}^{\varepsilon} \Phi$ and $M_{i}^{\varepsilon}, i \in\{1, \cdots, n\}, \varepsilon \in\{-,+\}$.

1.4. Example 1. In [8] Nazarova and Roiter examine the particular case of one pair of rods. The classification of representations in [7], [4], and [9] can be reduced to that of bushes.

Example 2. Representations of $\tilde{A_{n}}$. We illustrate the general construction with the following example: 


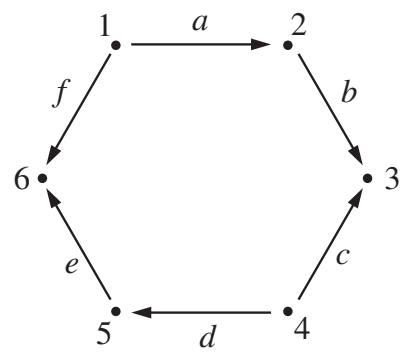

Set $S_{1}^{-}=\left\{a^{-}\right\}, S_{1}^{+}=\left\{a^{+}\right\}, \cdots, S_{6}^{-}=\left\{f^{-}\right\}, S_{6}^{+}=\left\{f^{+}\right\}$and equip $S=$ $\cup_{i=1}^{6}\left(S_{i}^{-} \cup S_{i}^{+}\right)=\left\{a^{-}, a^{+}, \cdots, f^{-}, f^{+}\right\}$with the equivalence relation $a^{-} \sim f^{-}$, $a^{+} \sim b^{-}, b^{+} \sim c^{+}, c^{-} \sim d^{-}, d^{+} \sim e^{-}, e^{+} \sim f^{+}$. Then rep $\tilde{A}_{6}$ is equivalent to $\operatorname{rep}\left(M^{-}, M^{+}\right)$, where $\left(M^{-}, M^{+}\right)$is the rodded tangle associated with the bush $S$ (see 1.3).

Example 3. Clannish algebras[2]: Let $Q$ be a quiver and $S p$ a set of loops in $Q$. The arrows in $S p$ are called "special" and the others "ordinary". Let further $R=Z \cup\left\{e^{2}-e: e \in S p\right\}$ be a set of "relations" of $Q$, where $Z$ consists of compositions $\mu \nu$ of ordinary arrows $\mu, \nu$. The algebra $A=k[Q] / R$, where $k[Q]$ denotes the algebra of the quiver $Q$, is called clannish if the following conditions hold:

(C1) At most two arrows start at each vertex, at most two stop;

(C2) For each ordinary arrow $a$, there is at most one arrow $b$ with $b a \notin Z$ and at most one $c$ with $a c \notin Z$;

(C3) Without real loss of generality, we further suppose that $R$ is minimal with respect to $(\mathrm{C} 2)$.

Examples. The algebras with the following data are clannish:

a)

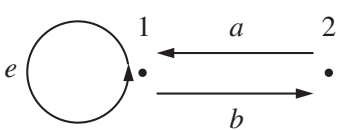

$$
S p=\{e\}
$$$$
Z=\{b a\}
$$

b)

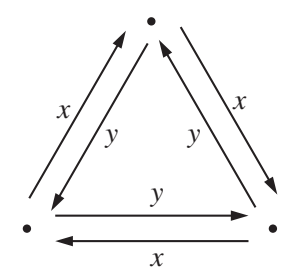

$S p=\emptyset$

$Z=\{$ all $x y, y x\}$ c)

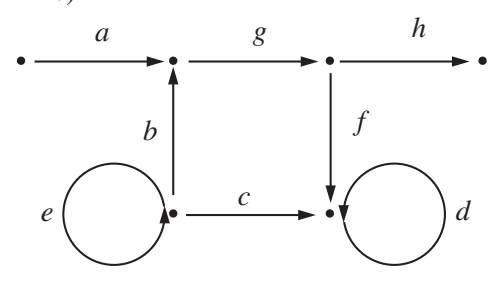

$$
S p=\{e\}
$$$$
Z=\left\{b a, c b, f c, d^{2}, h g\right\}
$$

With each clannish algebra $k[Q] / R$ we will associate a tangle. 
For the sake of simplicity, we identify the set $Q_{v}$ of vertices with $\{1,2, \cdots, n\}$. We further set $x^{-}=(x,-), x^{+}=(x,+)$ whenever $x$ is a vertex or an ordinary arrow. To each $i \in Q_{v}$, we then attach a set $A_{i}$ consisting of $i^{-}$and $i^{+}$, of special loops at $i$, and of all $x^{-}$(resp. $x^{+}$) where $x$ is an ordinary arrow starting (resp. stopping) at $i$. Finally, we construct two disjoint rods $S_{i}^{-}, S_{i}^{+}$such that:

a) $i^{-} \in S_{i}^{-}, i^{+} \in S_{i}^{+}$and $A_{i}=S_{i}^{-} \cup S_{i}^{+}$,

b) Each $S_{i}^{\varepsilon}$ has one of the following forms:

1) $\left\{i^{\varepsilon} \preccurlyeq e\right\}$, where $e$ is a special loop at $i$,

2) $\left\{a^{-}<i^{\varepsilon}\right\}$, where $a$ is an ordinary arrow starting at $i$,

3) $\left\{i^{\varepsilon}<b^{+}\right\}$, where $b$ is an ordinary arrow stopping at $i$,

4) $\left\{a^{-}<i^{\varepsilon}<b^{+}\right\}$, where $a$ (resp. b) is an ordinary arrow starting (resp. stopping) at $i$ and $a b \in Z$.

Of course, if $A_{i} \neq\left\{i^{-}, i^{+}\right\}$, there are exactly two possible choices for $S_{i}^{-}$and $S_{i}^{+}$. For instance, in case
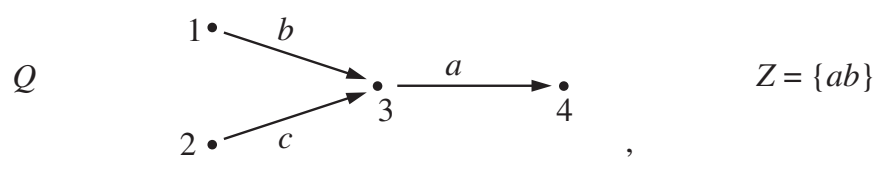

we obtain $S_{3}^{-}=\left\{a^{-}<3^{-}<b^{+}\right\}, S_{3}^{+}=\left\{3^{+}<c^{+}\right\}$, or reversely, $S_{3}^{-}=\left\{3^{-}<\right.$ $\left.c^{+}\right\}, S_{3}^{+}=\left\{a^{-}<3^{+}<b^{+}\right\}$.

We equip $S=\cup_{i=1}^{n}\left(S_{i}^{-} \cup S_{i}^{+}\right)$with an equivalence relation such that $a^{-} \sim a^{+}$ for each ordinary arrow $a$.

We denote by $\left(M^{-}, M^{+}\right)$the tangle associated with $S$ and by $\operatorname{rep}_{b}\left(M^{-}, M^{+}\right)$ the full subcategory of $\operatorname{rep}\left(M^{-}, M^{+}\right)$fromed by representations $\left(X ; f_{1}, \cdots, f_{n}\right)$ such that all $f_{i}$ are bijective.

Proposition. $\operatorname{rep}(Q, R)$ is equivalent to $\operatorname{rep}_{b}\left(M^{-}, M^{+}\right)$.

Proof. For each arrow $a \notin S p$ with $a^{-} \in S_{i}^{\varepsilon}$ and $a^{+} \in S_{j}^{\eta}$, and each $X \in \mathcal{A}$, we denote by $\xi_{X}^{a}$ the canonical isomorphism

$$
M_{i}^{\varepsilon}(X) / \mathcal{R} M_{i}^{\varepsilon}(X) \rightarrow \mathcal{R}^{h_{j}^{\eta}} M_{j}^{\eta}(X)
$$

where $h_{j}^{\eta}=1$ (resp. 2) if $S_{j}^{\eta}$ consists of 2 (resp. 3) elements.

For each $e \in S p$ with $e \in S_{i}^{\varepsilon}$, we denote by $J_{i}^{\varepsilon}$ and $L_{i}^{\varepsilon}$ the simple submodules of $M_{i}^{\varepsilon}$ supported by $i^{\varepsilon}$ and $e$ respectively.

With each object $\left(X ; f_{1}, \cdots, f_{n}\right)$ in $\operatorname{rep}_{b}\left(M^{-}, M^{+}\right)$we attach an object $V=$ $(V(i), V(\alpha))$ in $\operatorname{rep}(Q, R)$ as follows:

1) $V(i)=M_{i}^{+}(X), i=1, \cdots, n$.

2) For each arrow $\alpha: i \longrightarrow j$, in order to define $V(\alpha): M_{i}^{+}(X) \rightarrow M_{j}^{+}(X)$ we consider two cases. 
Case 1. $\alpha \notin S p, \alpha^{-} \in S_{i}^{\varepsilon}, \alpha^{+} \in S_{j}^{\varepsilon}$. If $\varepsilon=\eta=+, V(\alpha)$ is the composition

$$
M_{i}^{+}(X) \stackrel{\mathrm{pr}}{\longrightarrow} M_{i}^{+}(X) / \mathcal{R} M_{i}^{+}(X) \stackrel{\xi_{X}^{\alpha}}{\longrightarrow} \mathcal{R}^{h_{j}^{+}} M_{j}^{+}(X) \stackrel{\mathrm{im}}{\longrightarrow} M_{j}^{+}(X) ;
$$

if $\varepsilon=+$ and $\eta=-, V(\alpha)$ is the composition

$$
M_{i}^{+}(X) \stackrel{\mathrm{pr}}{\longrightarrow} M_{i}^{+}(X) / \mathcal{R} M_{i}^{+}(X) \stackrel{\xi_{X}^{\alpha}}{\longrightarrow} \mathcal{R}^{h_{j}^{-}} M_{j}^{-}(X) \stackrel{\mathrm{im}}{\longrightarrow} M_{j}^{-}(X) \stackrel{f_{j}}{\longrightarrow} M_{j}^{+}(X)
$$

if $\varepsilon=-$ and $\eta=+, V(\alpha)$ is the composition

$$
M_{i}^{+}(X) \stackrel{f_{i}^{-1}}{\longrightarrow} M_{i}^{-}(X) \stackrel{\mathrm{pr}}{\longrightarrow} M_{i}^{-}(X) / \mathcal{R} M_{i}^{-}(X) \stackrel{\xi_{X}^{\alpha}}{\longrightarrow} \mathcal{R}^{h_{j}^{+}} M_{j}^{+}(X) \stackrel{\mathrm{im}}{\longrightarrow} M_{j}^{+}(X)
$$

if $\varepsilon=\eta=-, V(\alpha)$ is the composition

$$
\begin{aligned}
M_{i}^{+}(X) \stackrel{f_{i}^{-1}}{\longrightarrow} M_{i}^{-}(X) \stackrel{\mathrm{pr}}{\longrightarrow} M_{i}^{-}(X) / \mathcal{R} M_{i}^{-}(X) & \\
& \stackrel{\xi_{X}^{\alpha}}{\longrightarrow} \mathcal{R}^{h_{j}^{-}} M_{j}^{-}(X) \stackrel{\mathrm{im}}{\longrightarrow} M_{j}^{-}(X) \stackrel{f_{j}}{\longrightarrow} M_{j}^{+}(X) .
\end{aligned}
$$

(By pr we denote the canonical projection, by im the canonical immersion.)

Case 2. $\alpha \in S p$ and $\alpha \in S_{i}^{\varepsilon}$ for some $i \in\{1, \cdots, n\}, \varepsilon \in\{-,+\}$. If $\varepsilon=+$, $V(\alpha)$ is identified with

$$
0 \oplus \mathbb{1}_{L_{i}^{+}(X)}: M_{i}^{+}(X)=J_{i}^{+}(X) \oplus L_{i}^{+}(X) \longrightarrow J_{i}^{+}(X) \oplus L_{i}^{+}(X)=M_{i}^{+}(X) ;
$$

if $\varepsilon=-, V(\alpha)$ is the composition

$$
\begin{aligned}
M_{i}^{+}(X) \stackrel{f_{i}^{-1}}{\longrightarrow} & M_{i}^{-}(X)=J_{i}^{-}(X) \oplus L_{i}^{-}(X) \\
0 \oplus \mathbb{1}_{L_{i}^{-}(X)}^{\longrightarrow} & J_{i}^{-}(X) \oplus L_{i}^{-}(X)=M_{i}^{-}(X) \stackrel{f_{i}}{\longrightarrow} M_{i}^{+}(X) .
\end{aligned}
$$

Thus we obtain a functor

$$
\begin{aligned}
F: \operatorname{rep}_{b}\left(M^{-}, M^{+}\right) & \longrightarrow \operatorname{rep}(Q, R) \\
\left(X ; f_{1}, \cdots, f_{n}\right) & \longmapsto V=(V(i), V(\alpha))
\end{aligned}
$$

which maps a morphism $\mu:\left(X ; f_{1}, \cdots, f_{n}\right) \longrightarrow\left(X^{\prime} ; f_{1}^{\prime}, \cdots, f_{n}^{\prime}\right)$ to the morphism $F(\mu):=\left(M_{i}^{+}(\mu)\right)_{i \in Q_{v}}$.

Since $\left(M^{-}, M^{+}\right)$is rodded and faithful, the functor $F$ is fully faithful.

Let $V=(V(i), V(\alpha)) \in \operatorname{rep}(Q, R)$. For each arrow $\alpha$ from $i$ to $j$, we set $K_{i}^{\alpha}=$ $\operatorname{ker} V(\alpha), I_{j}^{\alpha}=\operatorname{Im} V(\alpha)$ and denote by $V \overline{(\alpha)}$ the isomorphism $V(i) / \operatorname{ker} V(\alpha) \longrightarrow$ $\operatorname{Im} V(\alpha)$ induced by $V(\alpha)$.

In case $S_{i}^{\varepsilon}=\left\{i^{\varepsilon}\right\}$, we set $P_{i}^{\varepsilon}=V_{i}^{\varepsilon}=V(i)$ and denote by $\Phi_{i}^{\varepsilon}$ the identity $\mathbb{1}_{V(i)}$. 
In case $S_{i}^{\varepsilon}=\left\{i^{\varepsilon} \gtrless e\right\}$, we set $P_{i}^{\varepsilon}=K_{i}^{e}, V_{i}^{\varepsilon}=K_{i}^{e} \oplus I_{i}^{e}$, and denote by $\Phi_{i}^{\varepsilon}$ the canonical isomorphism $K_{i}^{e} \oplus I_{i}^{e} \longrightarrow V(i),(x, y) \longmapsto x+y$.

In case $S_{i}^{\varepsilon}=\left\{a^{-}<i^{\varepsilon}\right\}$, we set $P_{i}^{\varepsilon}=K_{i}^{a}, V_{i}^{\varepsilon}=I_{j}^{a} \oplus K_{i}^{a}$, and choose a section $t_{i}$ of the canonical projection $V(i) \longrightarrow V(i) / K_{i}^{a}$, we then denote by $\Phi_{i}^{\varepsilon}$ the composition

$$
V_{i}^{\varepsilon}=I_{j}^{a} \oplus K_{i}^{a} \stackrel{(V \overline{(a)})^{-1} \oplus \mathbb{1}_{K_{i}^{a}}}{\longrightarrow} V(i) / K_{i}^{a} \oplus K_{i}^{a} \stackrel{\left[t_{i} \text { im }\right]}{\longrightarrow} V(i) .
$$

In case $S_{i}^{\varepsilon}=\left\{i^{\varepsilon}<b^{+}\right\}$, we set $P_{i}^{\varepsilon}=V(i) / I_{i}^{b}, V_{i}^{\varepsilon}=V(i) / I_{i}^{b} \oplus I_{i}^{b}$, and choose a section $s_{i}$ of the canonical projection $V(i) \longrightarrow V(i) / I_{i}^{b}$, we then denote by $\Phi_{i}^{\varepsilon}$ the isomorphism $\left[s_{i} \mathrm{im}\right]: V_{i}^{\varepsilon}=V(i) / I_{i}^{b} \oplus I_{i}^{b} \longrightarrow V(i)$.

In case $S_{i}^{\varepsilon}=\left\{a^{-}<i^{\varepsilon}<b^{+}\right\}$, we set $P_{i}^{\varepsilon}=K_{i}^{a} / I_{i}^{b}, V_{i}^{\varepsilon}=I_{j}^{a} \oplus K_{i}^{a} / I_{i}^{b} \oplus I_{i}^{b}$, and choose a section $u_{i}$ of the canonical projection $K_{i}^{a} \longrightarrow K_{i}^{a} / I_{i}^{b}$ and a section $v_{i}$ of the canonical projection $V(i) \longrightarrow V(i) / K_{i}^{a}$, we then denote by $\Phi_{i}^{\varepsilon}$ the composition

$$
\begin{aligned}
& V_{i}^{\varepsilon}=I_{j}^{a} \oplus K_{i}^{a} / I_{i}^{b} \oplus I_{i}^{b} \stackrel{(V \overline{(a)})^{-1} \oplus \mathbb{1} \oplus \mathbb{1}}{\longrightarrow} V(i) / K_{i}^{a} \oplus K_{i}^{a} / I_{i}^{b} \oplus I_{i}^{b} \\
& \stackrel{\mathbb{1} \oplus\left[u_{i} \mathrm{im}\right]}{\longrightarrow} V(i) / K_{i}^{a} \oplus K_{i}^{a} \stackrel{\left[v_{i} \mathrm{im}\right]}{\longrightarrow} V(i) .
\end{aligned}
$$

Finally, we set

$$
X=\bigoplus_{a \in Q_{a} \backslash S p} \bar{a} \otimes I_{j}^{a} \oplus\left(\bigoplus_{e \in S p} e \otimes I_{i}^{e}\right) \oplus\left(\bigoplus_{i^{\varepsilon} \in S_{i}^{\varepsilon}} i^{\varepsilon} \otimes P_{i}^{\varepsilon}\right) \in \mathcal{A}
$$

and denote by $f_{i}$ the composition

$$
M_{i}^{-}(X) \stackrel{\text { can. }}{\cong} V_{i}^{-} \stackrel{\Phi_{i}^{-}}{\longrightarrow} V(i) \stackrel{\left(\Phi_{i}^{+}\right)^{-1}}{\longrightarrow} V_{i}^{+} \stackrel{\Psi_{i}}{\cong} M_{i}^{+}(X),
$$

where $\bar{a}$ denotes the equivalence class of $a$.

Thus we obtain an object $\left(X ; f_{1}, \cdots, f_{n}\right)$ in $\operatorname{rep}_{b}\left(M^{-}, M^{+}\right)$. By $\Psi_{i}$ we denote the canonical isomorphism $V_{i}^{+} \rightarrow M_{i}^{+}(X)$. Then $\left(\Psi_{i}\left(\Phi_{i}^{+}\right)^{-1}\right)_{i \in Q_{v}}$ defines an isomorphism from $V=(V(i), V(\alpha))$ to $F\left(X ; f_{1}, \cdots, f_{n}\right)$. Therefore, $F$ hits each isoclass in $\operatorname{rep}(Q, R)$.

1.5. Remark. With each tangle $\left(M^{-}, M^{+}\right)$over $\mathcal{A}$ we can associate as follows a tangle $\left(\bar{M}^{-}, \bar{M}^{+}\right)$over a new aggregate $\overline{\mathcal{A}}$.

Let $\mathcal{\mathcal { S }}$ denote the spectroid obtained from the spectroid $\mathcal{S}$ of $\mathcal{A}$ by adding objects $s_{i}$ and $t_{i}$ for $i \in\{1, \cdots, n\}$, whose spaces of radical morphisms $\mathcal{R}_{\overline{\mathcal{S}}}(x, y)$ are $\mathcal{R}_{\mathcal{S}}(x, y)$ if $x, y \in \mathcal{S}, \operatorname{Hom}_{k}\left(k, M_{i}^{-}(y)\right)$ if $y \in \mathcal{S}, x=s_{i}, \operatorname{Hom}_{k}\left(M_{i}^{+}(x), k\right)$ if $x \in \mathcal{S}, y=t_{i}$, and 0 otherwise. The composition $g \circ f$ of $f \in \mathcal{R}_{\overline{\mathcal{S}}}(x, y)$ and $g \in \mathcal{R}_{\overline{\mathcal{S}}}(y, z)$ is $g f$ if $x, y, z \in \mathcal{S}, M_{i}^{-}(g) f$ if $y, z \in \mathcal{S}, x=s_{i}, g M_{i}^{+}(f)$ if $x, y \in \mathcal{S}, z=t_{i}$, and 0 in all the remaining cases. 
Let $\overline{\mathcal{A}}$ denote the additive hull of $\overline{\mathcal{S}}$ and $\bar{M}_{i}^{-}$(resp. $\bar{M}_{i}^{+}$) the module over $\overline{\mathcal{A}}$ such that the value $\bar{M}_{i}^{-}(x)\left(\right.$ resp. $\left.\bar{M}_{i}^{+}(x)\right)$ at $x \in \overline{\mathcal{S}}$ is $k$ if $x=s_{i}\left(\right.$ resp. $\left.x=t_{i}\right)$, is $M_{i}^{-}(x)$ (resp. $\left.M_{i}^{+}(x)\right)$ if $x \in \mathcal{S}$, and 0 otherwise. If $f \in \mathcal{R}_{\overline{\mathcal{S}}}(x, y)$, the morphism $\bar{M}_{i}^{-}(f) \in \operatorname{Hom}_{k}\left(\bar{M}_{i}^{-}(x), \bar{M}_{i}^{-}(y)\right)\left(\operatorname{resp} . \bar{M}_{i}^{+}(f) \in \operatorname{Hom}_{k}\left(\bar{M}_{i}^{+}(x), \bar{M}_{i}^{+}(y)\right)\right)$ is $M_{i}^{-}(f)$ (resp. $\left.M_{i}^{+}(f)\right)$ if $x, y \in \mathcal{S}$, is $f$ if $x=s_{i}$ (resp. $y=t_{i}$ ), and 0 otherwise.

By the construction, the tangle $\left(\bar{M}^{-}, \bar{M}^{+}\right)$is rodded if so is $\left(M^{-}, M^{+}\right)$.

Let $\Psi: \overline{\mathcal{A}} \longrightarrow \mathcal{A}$ be the natural functor which maps $\bar{X} \in \overline{\mathcal{A}}$ onto the "largest summand" $\Psi(\bar{X})$ belonging to $\mathcal{A}$. Then $M_{i}^{-}$provides a submodule $M_{i}^{-} \Psi$ of $\bar{M}_{i}^{-}$, $M_{i}^{+}$provides a subquotient $M_{i}^{+} \Psi$ of $\bar{M}_{i}^{+}, i \in\{1, \cdots, n\}$, and $\Psi$ gives rise to a functor

$$
\begin{aligned}
F: \operatorname{rep}_{b}\left(\bar{M}^{-}, \bar{M}^{+}\right) & \longrightarrow \operatorname{rep}\left(M^{-}, M^{+}\right) \\
\left(\bar{X} ; \bar{f}_{1}, \cdots, \bar{f}_{n}\right) & \longmapsto\left(\Psi(\bar{X}) ; f_{1}, \cdots, f_{n}\right),
\end{aligned}
$$

where $f_{i}$ is the composition

$$
M_{i}^{-}(\Psi(\bar{X}))=M_{i}^{-} \Psi(\bar{X}) \stackrel{\mathrm{im}}{\longrightarrow} \bar{M}_{i}^{-}(\bar{X}) \stackrel{\bar{f}_{i}}{\longrightarrow} \bar{M}_{i}^{+}(\bar{X}) \stackrel{\mathrm{pr}}{\longrightarrow} M_{i}^{+} \Psi(\bar{X})=M_{i}^{+}(\Psi(\bar{X})) .
$$

Proposition. The functor $F$ is quasisurjective, and the indecomposables annihilated by $F$ are those isomorphic to $\left(s_{i} \oplus t_{i} ; 0, \cdots, 0, \mathbb{1}, 0, \cdots, 0\right), i=1, \cdots, n$.

Proof. Let $\left(X ; f_{1}, \cdots, f_{n}\right)$ be an object in $\operatorname{rep}\left(M^{-}, M^{+}\right)$. Consider the sequence

$$
K_{i}:=\operatorname{ker} f_{i} \stackrel{\mathrm{im}}{\longrightarrow} M_{i}^{-}(X) \stackrel{f_{i}}{\longrightarrow} M_{i}^{+}(X) \stackrel{\mathrm{pr}}{\longrightarrow} \operatorname{Coker} f_{i}=: C_{i} .
$$

Choose a retraction $p_{i}$ of the canonical immersion and a section $\mu_{i}$ of the canonical projection above, then

$$
\left[\begin{array}{cc}
\mu_{i} & f_{i} \\
0 & p_{i}
\end{array}\right]: C_{i} \oplus M_{i}^{-}(X) \longrightarrow M_{i}^{+}(X) \oplus K_{i}
$$

is bijective.

Set $\bar{X}=\left(\oplus_{i=1}^{n} s_{i} \otimes C_{i}\right) \oplus X \oplus\left(\oplus_{i=1}^{n} t_{i} \otimes K_{i}\right) \in \overline{\mathcal{A}}$, and denote by $\bar{f}_{i}$ the composition

$$
\bar{M}_{i}^{-}(\bar{X}) \stackrel{\operatorname{can} .}{\cong} C_{i} \oplus M_{i}^{-}(X) \stackrel{\left[\begin{array}{cc}
\mu_{i} & f_{i} \\
0 & p_{i}
\end{array}\right]}{\longrightarrow} M_{i}^{+}(X) \oplus K_{i} \stackrel{\operatorname{can} .}{\cong} \bar{M}_{i}^{+}(\bar{X}) .
$$

Then $\left(\bar{X} ; \bar{f}_{1}, \cdots, \bar{f}_{n}\right) \in \operatorname{rep}_{b}\left(\bar{M}^{-}, \bar{M}^{+}\right)$and $F\left(\bar{X} ; \bar{f}_{1}, \cdots, \bar{f}_{n}\right) \cong\left(X ; f_{1}, \cdots, f_{n}\right)$.

It is not difficult to see that each $\left(\bar{X} ; \bar{f}_{1}, \cdots, \bar{f}_{n}\right) \in \operatorname{rep}_{b}\left(\bar{M}^{-}, \bar{M}^{+}\right)$is isomorphic to the direct sum of objects of the form $\left(s_{i} \oplus t_{i} ; 0, \cdots, 0, \mathbb{1}, 0, \cdots, 0\right)$ and of the 
form $\left.\bar{Y}=\left(\oplus_{i=1}^{n} s_{i} \otimes S_{i}\right) \oplus Y \oplus\left(\oplus_{i=1}^{n} t_{i} \otimes T_{i}\right) ; \bar{g}_{1}, \cdots, \bar{g}_{n}\right)$, where $S_{i}, T_{i} \in \bmod k$, $Y \in \mathcal{A}$, and $\bar{g}_{i}$ has the form

$$
\left[\begin{array}{cc}
a_{i} & g_{i} \\
0 & b_{i}
\end{array}\right]: S_{i} \oplus M_{i}^{-}(Y) \longrightarrow M_{i}^{+}(Y) \oplus T_{i}
$$

where $a_{i}, b_{i}$ and $g_{i}$ are $k$-linear maps.

Let $\left(\bar{X} ; \bar{f}_{1}, \cdots, \bar{f}_{n}\right)$ and $\left(\bar{X}^{\prime} ; \bar{f}_{1}^{\prime}, \cdots, \bar{f}_{n}^{\prime}\right)$ be objects in $\operatorname{rep}_{b}\left(\bar{M}^{-}, \bar{M}^{+}\right)$, and $\mu \in$ $\mathcal{A}\left(\Psi(\bar{X}), \Psi\left(\bar{X}^{\prime}\right)\right)$ a morphism from $F\left(\bar{X} ; \bar{f}_{1}, \cdots, \bar{f}_{n}\right)$ to $F\left(\bar{X}^{\prime} ; \bar{f}_{1}^{\prime}, \cdots, \bar{f}_{n}^{\prime}\right)$

Since $F$ annihilates $\left(s_{i} \oplus t_{i} ; 0, \cdots, 0, \mathbb{1}, 0, \cdots, 0\right)$, we may assume that $\bar{X}$ and $\bar{X}^{\prime}$ have respectively the forms $\left(\oplus_{i=1}^{n} s_{i} \otimes S_{i}\right) \oplus X \oplus\left(\oplus_{i=1}^{n} t_{i} \otimes T_{i}\right)$ and $\left(\oplus_{i=1}^{n} s_{i} \otimes\right.$ $\left.S_{i}^{\prime}\right) \oplus X^{\prime} \oplus\left(\oplus_{i=1}^{n} t_{i} \otimes T_{i}^{\prime}\right)$, where $S_{i}, T_{i}, S_{i}^{\prime}, T_{i}^{\prime} \in \bmod k$ and $X, X^{\prime} \in \mathcal{A}$, and that $\bar{f}_{i}$ and $\bar{f}_{i}^{\prime}$ are of the forms:

$$
\begin{gathered}
\bar{f}_{i}=\left[\begin{array}{cc}
a_{i} & f_{i} \\
0 & b_{i}
\end{array}\right]: S_{i} \oplus M_{i}^{-}(X) \longrightarrow M_{i}^{+}(X) \oplus T_{i}, \\
\bar{f}_{i}^{\prime}=\left[\begin{array}{cc}
a_{i}^{\prime} & f_{i}^{\prime} \\
0 & b_{i}^{\prime}
\end{array}\right]: S_{i}^{\prime} \oplus M_{i}^{-}\left(X^{\prime}\right) \longrightarrow M_{i}^{+}\left(X^{\prime}\right) \oplus T_{i}^{\prime},
\end{gathered}
$$

where $a_{i}, b_{i}, f_{i}, a_{i}^{\prime}, b_{i}^{\prime}$ and $f_{i}^{\prime}$ are $k$-linear maps, $i \in\{1, \cdots, n\}$.

Thus $F\left(\bar{X} ; \bar{f}_{1}, \cdots, \bar{f}_{n}\right)=\left(X ; f_{1}, \cdots, f_{n}\right)$ and $F\left(\bar{X}^{\prime} ; \bar{f}_{1}^{\prime}, \cdots, \bar{f}_{n}^{\prime}\right)=\left(X^{\prime} ; f_{1}^{\prime}, \cdots\right.$, $\left.f_{n}^{\prime}\right)$

Consider the following commutative diagram

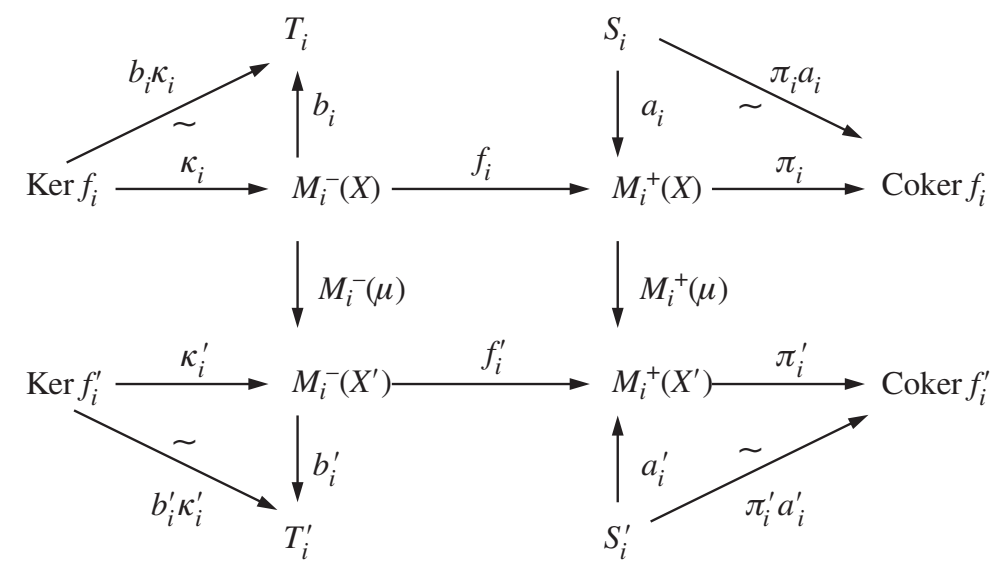

where $\kappa_{i}$ and $\kappa_{i}^{\prime}$ denote the canonical immersions, $\pi_{i}$ and $\pi_{i}^{\prime}$ the canonical projections.

The bijectivity of $\bar{f}_{i}$ and $\bar{f}_{i}^{\prime}$ implies that $b_{i} \kappa_{i}, b_{i}^{\prime} \kappa_{i}^{\prime}, \pi_{i} a_{i}$ and $\pi_{i}^{\prime} a_{i}^{\prime}$ are bijective. Set $u_{i}=\left(\pi_{i}^{\prime} a_{i}^{\prime}\right)^{-1} \pi_{i}^{\prime} M_{i}^{+}(\mu) a_{i}$ and $v_{i}=b_{i}^{\prime} M_{i}^{-}(\mu) \kappa_{i}\left(b_{i} \kappa_{i}\right)^{-1}$. It is easy to see that there exist a $w_{i}: S_{i} \longrightarrow M_{i}^{-}\left(X^{\prime}\right)$ and a $z_{i}: M_{i}^{+}(X) \longrightarrow T_{i}^{\prime}$ such that $z_{i} a_{i}=0$, 
$b_{i}^{\prime} w_{i}=0, M_{i}^{+}(\mu) a_{i}=a_{i}^{\prime} u_{i}+f_{i}^{\prime} w_{i}$, and $b_{i}^{\prime} M_{i}^{-}(\mu)=z_{i} f_{i}+v_{i} b_{i}$, i.e. the following square commutes.

$$
\begin{aligned}
& {\left[\begin{array}{ll}
a_{i} & f_{i} \\
0 & b_{i}
\end{array}\right]} \\
& S_{i} \oplus M_{i}^{-}(X) \stackrel{\longrightarrow}{\longrightarrow} M_{i}^{+}(X) \oplus T_{i} \\
& {\left.\left[\begin{array}{cc}
u_{i} & 0 \\
w_{i} & M_{i}^{-}(\mu)
\end{array}\right]\right|_{S_{i}^{\prime} \oplus M_{i}^{-}\left(X^{\prime}\right) \stackrel{\left[\begin{array}{cc}
a_{i}^{\prime} & f_{i}^{\prime} \\
0 & b_{i}^{\prime}
\end{array}\right]}{\longrightarrow} M_{i}^{+}(X) \oplus T_{i}^{\prime}}\left[\begin{array}{cc}
M_{i}^{+}(\mu) & 0 \\
z_{i} & v_{i}
\end{array}\right]}
\end{aligned}
$$

Set

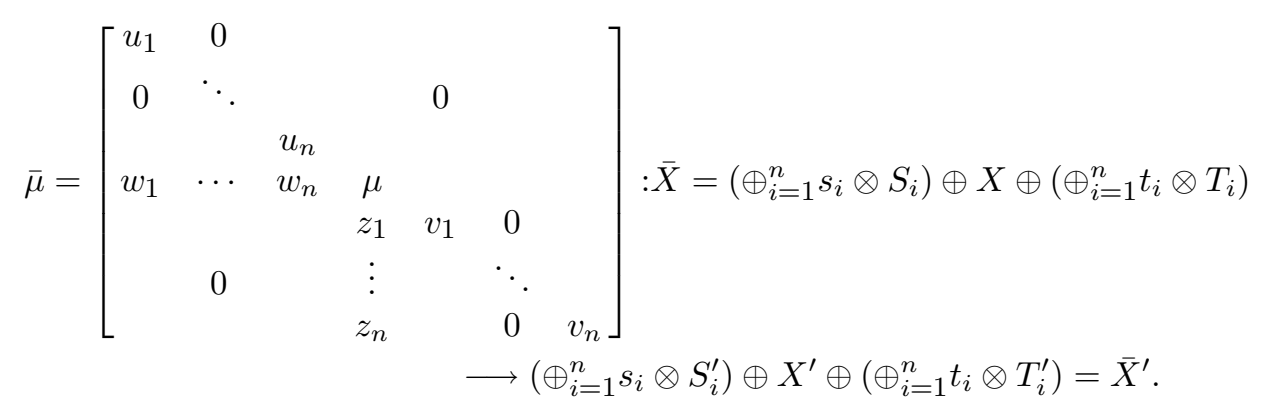

Then $F(\bar{\mu})=\mu$, that is, $F$ is full.

This finishes the proof of the proposition.

\section{The classification}

2.1. Terminology. Let $S=\left(S_{1}^{-}, \cdots, S_{n}^{-} ; S_{1}^{+}, \cdots, S_{n}^{+} ; \sim\right)$ be a bush.

In the sequel, we write $x \wedge y$ if $x$ and $y$ belong to the same rod and are incomparable, and we write $x \mid y$ if $(x, y) \in \cup_{i=1}^{n}\left(\left(S_{i}^{-} \times S_{i}^{+}\right) \cup\left(S_{i}^{+} \times S_{i}^{-}\right)\right)$. We further set

$$
\begin{aligned}
x^{\sim} & = \begin{cases}y & \text { if } y \sim x \text { and } y \neq x \\
x & \text { if the equivalence class of } x \text { contains only } x\end{cases} \\
x^{\wedge} & = \begin{cases}y & \text { if } y \wedge x \\
x & \text { if } x \text { is comparable with all points of its rod }\end{cases} \\
\text { and } \quad x^{*} & =\left(x^{\sim}\right)^{\wedge} .
\end{aligned}
$$


We call catenation of $S$ a sequence $w=w_{1} w_{2} \cdots w_{t}$ of points of $S$ such that $w_{i}^{*} \mid w_{i+1}$ for all $i<t$. The reverse catenation is the sequence $w^{*}=w_{t}^{*} \cdots w_{2}^{*} w_{1}^{*}$. Two catenations $w=w_{1} w_{2} \cdots w_{s}$ and $w^{\prime}=w_{1}^{\prime} w_{2}^{\prime} \cdots w_{t}^{\prime}$ are called equivalent if $s=t$ and $w_{i}^{\prime}=w_{i}$ or $w_{i}^{\wedge}$ for all $1 \leq i \leq s$. For each catenation $w$, we then denote by $[w]$ the equivalence class of $w$. Then the set of equivalence classes of all catenations of $S$ is equipped with an order relation such that

$$
\begin{gathered}
{[v]=\left[v_{1} \cdots v_{s}\right]<\left[w_{1} \cdots w_{t}\right]=[w]} \\
\Longleftrightarrow\left\{\begin{array}{c}
\text { if } w=v^{\prime} w^{\prime},\left[v^{\prime}\right]=[v], w_{1}^{\prime}=w_{s+1} \in S^{-} \\
\text {or } \quad \text { if } v=w^{\prime} v^{\prime},\left[w^{\prime}\right]=[w], v_{1}^{\prime}=v_{t+1} \in S^{+} \\
\text {or } \quad \text { if } v=u x v^{\prime}, w=u^{\prime} y w^{\prime},[u]=\left[u^{\prime}\right], x<y .
\end{array}\right.
\end{gathered}
$$

The equivalence classes of catenations which start in a fixed rod are pairwise comparable.

2.2. From now onwards, we suppose that $S$ is complete, i.e. that $x \neq x^{*}$ for all $x \in S$. [This is no real restriction. Otherwise, we replace $S$ by a completed bush $S^{o}$ obtained from $S$ by adding new rods $S_{i x}^{-}=\left\{x^{o}\right\}, S_{i x}^{+}=\emptyset$ and by agreeing that $x \sim x^{o}$ for each point $x$ of $S$ such that $x=x^{\wedge}=x^{\sim}$. The new bush $S^{o}$ is complete, and $\operatorname{rep} S$ is equivalent to $\operatorname{rep} S^{\circ}$.]

If $S$ is complete, we attach a representation $\left(X_{w} ; f_{w 1}, \cdots, f_{w n}\right)$ of $S$ to each catenation $w=w_{1} w_{2} \cdots w_{t}$. First we set

$$
X_{w}=\widehat{w_{1}} \oplus \widehat{w_{2}} \oplus \cdots \oplus \widehat{w_{t}},
$$

where $\widehat{w_{i}}=\left\{w_{i}, w_{i}^{*}\right\} \in \mathcal{S}$ (=the spectroid attached to $S$ in 1.3) if $w_{i} \neq w_{i}^{\sim}$ and $\widehat{w_{i}}=\left\{w_{i}\right\} \oplus\left\{w_{i}^{*}\right\} \in \mathcal{A}$ (=the additive hull of $S$ ) if $w_{i} \neq w_{i}^{\wedge}$. Thus each term $x$ of the sequence $w_{1} w_{1}^{*} w_{2} w_{2}^{*} \cdots w_{t} w_{t}^{*}$ contributes a one-dimensional summand $k \underline{x}$ to the space $M_{i}^{\varepsilon}\left(X_{w}\right)$ associated with the $\operatorname{rod} S_{i}^{\varepsilon}$ containing $x$. Accordingly, $M_{i}^{-}\left(\bar{X}_{w}\right)$ and $M_{i}^{+}\left(X_{w}\right)$ have the form:

$$
(*) \quad\left\{\begin{array}{l}
M_{i}^{-}\left(X_{w}\right)=\oplus_{p} k \underline{w}_{p} \oplus \oplus_{l} k \underline{w}_{l}^{*} \\
M_{i}^{+}\left(X_{w}\right)=\oplus_{m} k \underline{w}_{m}^{*} \oplus \oplus_{q} k \underline{w}_{q}
\end{array}\right.
$$

where $p, q, l$ and $m$ are subjected to the conditions $w_{p}, w_{l}^{*} \in S_{i}^{-}$and $w_{q}, w_{m}^{*} \in$ $S_{i}^{+}$. The structure maps are defined as sums

$$
f_{w i}=\sum_{r} f_{w i r}: M_{i}^{-}\left(X_{w}\right) \longrightarrow M_{i}^{+}\left(X_{w}\right)
$$

where each $r>1$ satisfying $w_{r} \in S_{i}^{-} \cup S_{i}^{+}$provides a contribution

$$
f_{w i r}=h_{w i r} g_{w i r}: M_{i}^{-}\left(X_{w}\right) \stackrel{g_{w i r}}{\longrightarrow} k \stackrel{h_{w i r}}{\longrightarrow} M_{i}^{+}\left(X_{w}\right) .
$$


To define the factors $g_{w i r}$ and $h_{w i r}$, we distinguish two cases:

1) Case $w_{r-1}^{*} \in S_{i}^{-}, w_{r} \in S_{i}^{+}$. Construction of $g_{w i r}$ : If $w_{r-1}=w_{r-1}^{\wedge}$, we set $g_{w i r}\left(\underline{w}_{r-1}^{*}\right)=1$ and let $g_{w i r}$ vanish on the remaining basis vectors.

If $w_{r-1} \neq w_{r-1}^{\wedge}$, the map $g_{w i r}$ is the composition

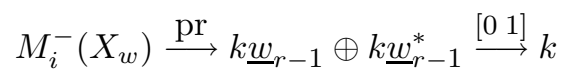

provided $\left[w_{r-2}^{*} \cdots w_{1}^{*}\right] \leq\left[w_{r} \cdots w_{t}\right]$. Otherwise, it is the composition

$$
M_{i}^{-}\left(X_{w}\right) \stackrel{\mathrm{pr}}{\longrightarrow} k \underline{w}_{r-1} \oplus k \underline{w}_{r-1}^{*} \stackrel{[1}{\longrightarrow} k
$$

(By pr we denote the projection which annihilates the basis vectors $\neq \underline{w}_{r-1}, \underline{w}_{r-1}^{*}$.)

Construction of $h_{w i r}$ : If $w_{r}=w_{r}^{\wedge}$, we define $h_{w i r}(1)=\underline{w}_{r}$. If $w_{r} \neq w_{r}^{\wedge}, \underline{h}_{w i r}$ is the composition

$$
k \stackrel{[10]^{T}}{\longrightarrow} k \underline{w}_{r} \oplus k \underline{w}_{r}^{*} \stackrel{\operatorname{im}}{\longrightarrow} M_{i}^{+}\left(X_{w}\right)
$$

provided $\left[w_{r-1}^{*} \cdots w_{1}^{*}\right] \leq\left[w_{r+1} \cdots w_{t}\right]$. Otherwise, it is the composition

$$
k \stackrel{[11]^{T}}{\longrightarrow} k \underline{w}_{r} \oplus k \underline{w}_{r}^{*} \stackrel{i m}{\longrightarrow} M_{i}^{+}\left(X_{w}\right)
$$

(By im we denote the canonical immersion.)

2) Case $w_{r-1}^{*} \in S_{i}^{+}, w_{r} \in S_{i}^{-}$. Construction of $g_{w i r}$ : If $w_{r}=w_{r}^{\wedge}$, we set $g_{w i r}\left(\underline{w}_{r}\right)=1$ and let $g_{w i r}$ vanish on the remaining basis vectors. If $w_{r} \neq w_{r}^{\wedge}$, the map $g_{w i r}$ is the composition

$$
M_{i}^{-}\left(X_{w}\right) \stackrel{\mathrm{pr}}{\longrightarrow} k \underline{w}_{r} \oplus k \underline{w}_{r}^{*} \stackrel{[10]}{\longrightarrow} k
$$

provided $\left[w_{r-1}^{*} \cdots w_{1}^{*}\right]>\left[w_{r+1} \cdots w_{t}\right]$. Otherwise, $\left[\begin{array}{ll}0 & 1\end{array}\right]$ is replaced by $\left[\begin{array}{ll}1 & 1\end{array}\right]$.

Construction of $h_{w i r}$ : If $w_{r-1}=w_{r-1}^{\wedge}$, we define $h_{w i r}(1)=\underline{w}_{r-1}^{*}$. If $w_{r-1} \neq$ $w_{r-1}^{\wedge}, h_{w i r}$ is the composition

$$
k \stackrel{[01]^{T}}{\longrightarrow} k \underline{w}_{r-1} \oplus k \underline{w}_{r-1}^{*} \stackrel{\operatorname{im}}{\longrightarrow} M_{i}^{+}\left(X_{w}\right)
$$

provided $\left[w_{r-2}^{*} \cdots w_{1}^{*}\right]>\left[w_{r} \cdots w_{t}\right]$. Otherwise, $\left[\begin{array}{ll}0 & 1\end{array}\right]^{T}$ is replaced by $\left[\begin{array}{ll}1 & 1\end{array}\right]^{T}$.

2.3. Example. The clannish algebra $k[Q] /\left(b a, e^{2}-e\right)$, where $Q$ denotes the quiver

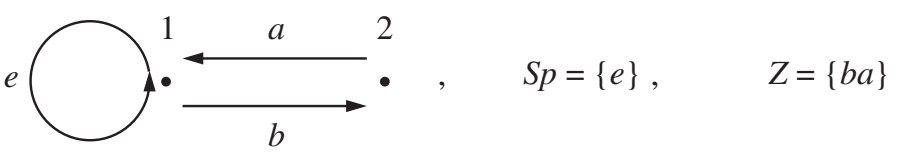


gives rise to the (non-completed) bush

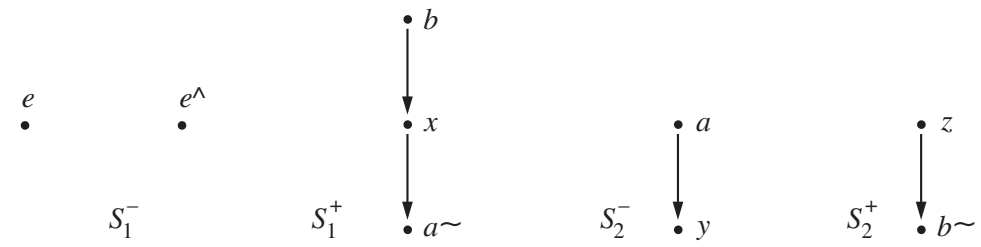

As a typical example, we choose the catenation $w=y^{\sim} b^{\sim}$ ebaex of the completed bush. The maps $f_{w i}$ then behave as follows:
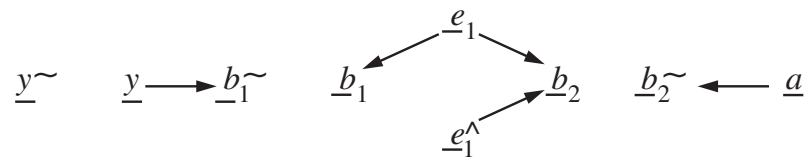

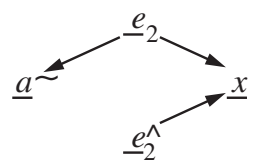

$\underline{x} \sim$

The matrices of the representation of the non-completed bush associated with $w$-or, more precisely, the matrices of the linear maps $f_{w 1}$ and $f_{w 2}$-are displayed as follows:

$\begin{array}{cc}e & e^{\wedge} \\ b\left[\begin{array}{cc|cc}1 & 0 & 0 & 0 \\ 1 & 0 & 1 & 0 \\ a^{\sim} & z & a \\ \hline 0 & 1 & 0 & 1 \\ \hline 0 & 1 & 0 & 0\end{array}\right] & b^{\sim}\left[\begin{array}{c|c} & \\ \hline 1 & 0 \\ 0 & 1\end{array}\right]\end{array}$

Similarly, the maps $f_{v i}$ of the representation associated with the catenation $v=x^{\sim}$ ebaea $\sim b^{\sim} e^{\wedge} x$ are

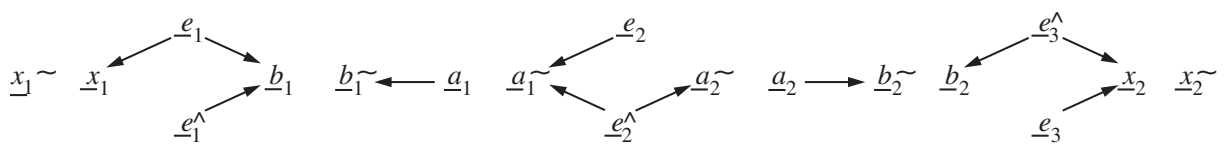

2.4. In the first example considered above, the catenation $w$ is asymmetric, i.e. $[w] \neq\left[w^{*}\right]$. The matrices of the representation associated with $w^{*}$ are

$\begin{array}{cc}e & e^{\wedge} \\ b\left[\begin{array}{cc|cc}0 & 1 & 0 & 1 \\ 0 & 1 & 0 & 0 \\ a^{\sim} & y & a \\ \hline 1 & 0 & 1 & 0 \\ \hline 1 & 0 & 0 & 0\end{array}\right] & z\left[\begin{array}{c|c} & \\ \hline 0 & 1 \\ 1 & 0\end{array}\right]\end{array}$


By "permissible" row and column transformations, these matrices can obviously be converted into the matrices associated with $w$. The representations associated with $w$ and $w^{*}$ are therefore isomorphic.

In general, we choose a set $\Omega_{1}$ of asymmetric catenations which contains one representative of each class $[w] \coprod\left[w^{*}\right]$ of asymmetric catenations. For each $w \in$ $\Omega_{1}$, we denote by $R(w)$ the corresponding representation of $S$. Representations isomorphic to such an $R(w)$ will be called asymmetric strings.

In the second example of 2.3 , the catenation $v$ is symmetric, i.e. $[v]=\left[v^{*}\right]$. In this case, the representation associated with $v$ is clearly the direct sum of two representations $R(v, 0)$ and $R(v, 1)$.

Of course, this is a general fact (This fact will be shown in Section 4). For each symmetric catenation $v$, the associated representation in 2.2 decomposes into the direct sum of two representations $R(v, 0)$ and $R(v, 1)$. These representations are indexed by $\Omega_{2} \times\{0,1\}$, where $\Omega_{2}$ denotes the set of symmetric catenations which contains one representative of each class $[w]$ of symmetric catenations. Representations of $S$ isomorphic to some $R(v, i),(v, i) \in \Omega_{2} \times\{0,1\}$, will be called dimidiate strings.

2.5. Besides finite catenations, we consider periodic catenations. These are sequences $u=\left(u_{i}\right)_{i \in \mathbb{Z}}$ which satisfy $u_{i}^{*} \mid u_{i+1}$ for all $i \in \mathbb{Z}$ and admit a natural number $\pi \geq 1$ such that $u_{i+\pi}=u_{i}$ or $u_{i}^{\wedge}$ for all $i$. The smallest $\pi$ satisfying these conditions is the period of $u$. Each periodic catenation $u$ is consorted with a reverse $u^{*}$ such that $\left(u^{*}\right)_{i}=\left(u_{-i}\right)^{*}$ and with translates $u\{p\}$ such that $u\{p\}_{i}=u_{p+i}$. It is called symmetric if $\left[u^{*}\right]=[u\{p\}]$ for some $p$ and asymmetric if not.

To each asymmetric period catenation $u$ we shall attach a family of representations of $S$ which are indexed by the powers

$$
Q=P^{l}=X^{m l}-a_{1} X^{m l-1}-a_{2} X^{m l-2}-\cdots-a_{m l}, \quad l \geq 1
$$

of the irreducible unitary polynomials $P$ in one determinate $X$ with coefficients in $k$. The index set formed by the powers $Q=P^{l}$ with $P \neq X$ is denoted by $\mathcal{P}$. To each $Q \in \mathcal{P}$ we attach the invertible matrix

$$
A(Q)=\left[\begin{array}{ccccc}
0 & 0 & \ldots & 0 & a_{m l} \\
1 & 0 & \ldots & 0 & a_{m l-1} \\
\vdots & \ddots & \ddots & \vdots & \vdots \\
0 & 0 & \ddots & 0 & a_{2} \\
0 & 0 & \ldots & 1 & a_{1}
\end{array}\right]
$$

The representation $\left(Y_{u}^{Q} ; \xi_{u 1}^{Q}, \cdots, \xi_{u n}^{Q}\right)$ associated with an asymmetric periodic catenation $u$ of period $\pi$ and a polynomial $Q \in \mathcal{P}$ of degree $d$ is obtained as follows. First we consider the representation $\left(X_{w} ; f_{w 1}, \cdots, f_{w n}\right)$ attached to the catenation

$$
\begin{aligned}
w & =w_{1} w_{2} \cdots w_{\pi} w_{\pi+1} \cdots w_{2 \pi} w_{2 \pi+1} \cdots w_{3 \pi} \\
& =u_{-\pi} u_{-\pi+1} \cdots u_{-1} u_{0} u_{1} \cdots u_{\pi-1} u_{\pi} \cdots u_{2 \pi-1} .
\end{aligned}
$$


With the notations of 2.2 we then set

$$
Y_{u}^{Q}={\widehat{w_{\pi+1}}}^{d} \oplus{\widehat{w_{\pi+2}}}^{d} \oplus \cdots \oplus{\widehat{w_{2 \pi}}}^{d}={\widehat{u_{0}}}^{d} \oplus{\widehat{u_{1}}}^{d} \oplus \cdots \oplus{\widehat{u_{\pi-1}}}^{d}
$$

and thus obtain

$$
\begin{aligned}
& M_{i}^{-}\left(Y_{u}^{Q}\right)=\left(\oplus_{p} k \underline{u}_{p} \oplus \oplus_{l} k \underline{u}_{l}^{*}\right) \otimes_{k} k^{d} \\
& M_{i}^{+}\left(Y_{u}^{Q}\right)=\left(\oplus_{m} k \underline{u}_{m}^{*} \oplus \oplus_{q} k \underline{u}_{q}\right) \otimes_{k} k^{d}
\end{aligned}
$$

where $l, m, p$ and $q$ belong to $\{0,1, \cdots, \pi-1\}$ and satisfy $u_{p}, u_{l}^{*} \in S_{i}^{-}$and $u_{q}, u_{m}^{*} \in S_{i}^{+}$. The structure maps are defined as sums

$$
\xi_{u i}^{Q}=\lambda_{u i}^{Q}+\mu_{u i}^{Q}: M_{i}^{-}\left(Y_{u}^{Q}\right) \longrightarrow M_{i}^{+}\left(Y_{u}^{Q}\right),
$$

where $\lambda_{u i}^{Q}$ is the composition

$$
M_{i}^{-}\left(Y_{u}^{Q}\right) \stackrel{\mathrm{im}}{\longrightarrow} M_{i}^{-}\left(X_{w}\right) \otimes_{k} k^{d \stackrel{f_{w i} \otimes \mathbb{1}_{d}}{\longrightarrow}} M_{i}^{+}\left(X_{w}\right) \otimes_{k} k^{d} \stackrel{\mathrm{pr}}{\longrightarrow} M_{i}^{+}\left(Y_{u}^{Q}\right)
$$

(By im we denote the canonical immersion, and by pr the canonical projection.), and where $\mu_{u i}^{Q}$ is the composition

$$
\begin{aligned}
M_{i}^{-}\left(Y_{u}^{Q}\right) \stackrel{\mathrm{im}}{\longrightarrow} M_{i}^{-}\left(X_{w}\right) \otimes_{k} k^{d} \stackrel{g_{w i 2 \pi+1} \otimes A(Q)}{\longrightarrow} k \otimes_{k} k^{d} \stackrel{h_{w i \pi+1} \otimes \mathbb{1}_{d}}{\longrightarrow} M_{i}^{+}\left(X_{w}\right) \otimes_{k} k^{d} \\
\stackrel{\mathrm{pr}}{\longrightarrow} M_{i}^{+}\left(Y_{u}^{Q}\right)
\end{aligned}
$$

if $u_{0} \in S_{i}^{+}$;

$$
\begin{aligned}
M_{i}^{+}\left(Y_{u}^{Q}\right) \longleftarrow M_{i}^{+}\left(X_{w}\right) \otimes_{k} k^{d} \stackrel{h_{w i} \longleftarrow^{2 \pi+1} \mathbb{1}_{d}}{\longleftarrow} k \otimes_{k} k^{d g_{w i \pi+1} \otimes A}(Q)^{-1} & M_{i}^{-}\left(X_{w}\right) \otimes_{k} k^{d} \\
& \stackrel{\operatorname{im}}{\longleftarrow} M_{i}^{-}\left(Y_{u}^{Q}\right)
\end{aligned}
$$

if $u_{0} \in S_{i}^{-}$; and zero if $u_{0} \notin S_{i}^{-} \cup S_{i}^{+}$.

As a typical example, we consider the case where $S$ is as in 2.3 , and

$$
u_{0} u_{1} \cdots u_{\pi-1}=a e b a e^{\wedge} a^{\sim} b^{\sim} e b .
$$

The structure maps $g_{u i}^{Q}$ are then visualized by the following diagram

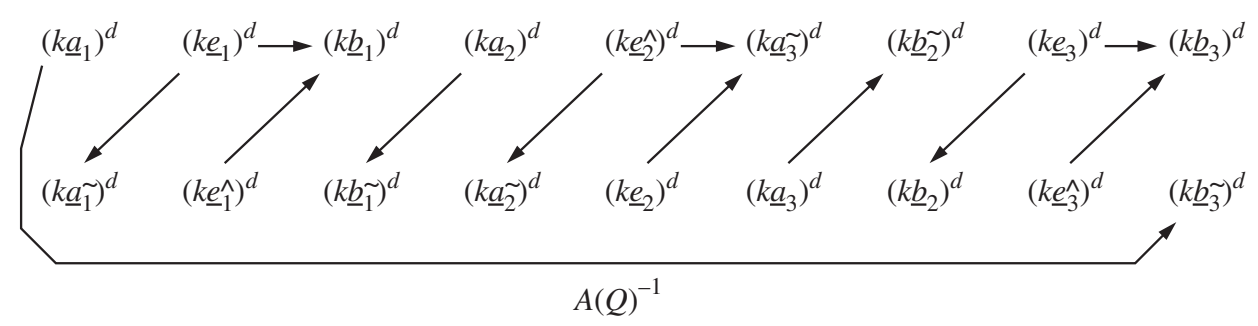


The corresponding matrices are

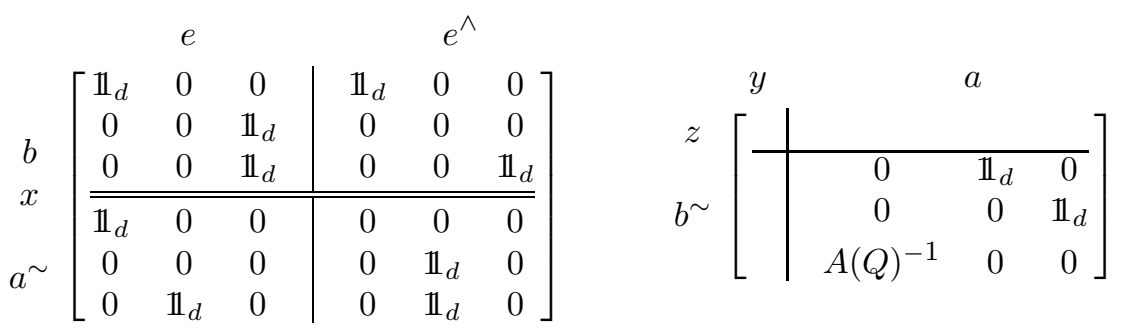

In view of the required classification, we now choose a set $\Omega_{3}$ of asymmetric periodic catenations which contains one representative of each class $\coprod_{p \in \mathbb{Z}}([u\{p\}]$ $\left.\coprod\left[u\{p\}^{*}\right]\right)$. For each $(u, Q) \in \Omega_{3} \times \mathcal{P}$, we denote the representation constructed above by $R(u, Q)$. A representation of $S$ isomorphic to such an $R(u, Q)$ will be called an asymmetric band.

2.6. We finally turn to the case of a symmetric periodic catenation $u$. It is easy to prove that $u_{0} u_{1} \cdots u_{\pi-1}$ then has the form

$$
u_{0} u_{1} \cdots u_{\pi-1}=a_{1} \cdots a_{r} e b_{r} \cdots b_{1} c_{1} \cdots c_{s} f d_{s} \cdots d_{1}
$$

where $\left[a_{r}^{*} \cdots a_{1}^{*}\right]=\left[b_{r} \cdots b_{1}\right],\left[c_{s}^{*} \cdots c_{1}^{*}\right]=\left[d_{s} \cdots d_{1}\right], e \neq e^{\wedge}$, and $f \neq f^{\wedge}$. Setting

$$
\begin{aligned}
w & =w_{1} w_{2} \cdots w_{\pi} w_{\pi+1} \cdots w_{2 \pi} w_{2 \pi+1} \cdots w_{3 \pi} \\
& =u_{-\pi} u_{-\pi+1} \cdots u_{-1} u_{0} u_{1} \cdots u_{\pi-1} u_{\pi} \cdots u_{2 \pi-1} .
\end{aligned}
$$

as in 2.5. We shall associate a representation $\left(Z_{u}^{K} ; \eta_{u 1}^{K}, \cdots, \eta_{u n}^{K}\right)$ with each matrix

$$
K=\left[\begin{array}{l|l}
A & B \\
\hline C & D
\end{array}\right] \in k^{\left(m+m^{\prime}\right) \times\left(l+l^{\prime}\right)}
$$

belonging to $\mathcal{Q}$. By $\mathcal{Q}$ we denote the set of the following matrices $(q \geq 0)$ :

1)

$\left[\begin{array}{c|c}0 & \mathbb{1}_{q+1} \\ \mathbb{1}_{q} & \\ \hline \mathbb{1}_{q} & \mathbb{1}_{q} 0\end{array}\right],\left[\begin{array}{c|c}\mathbb{1}_{q} 0 & \mathbb{1}_{q} \\ \hline \mathbb{1}_{q+1} & 0 \\ \mathbb{1}_{q}\end{array}\right],\left[\begin{array}{l|l}\mathbb{1}_{q+1} & \mathbb{1}_{q+1} \\ \hline \mathbb{1}_{q+1} & J_{q+1}\end{array}\right],\left[\begin{array}{c|c}J_{q+1} & \mathbb{1}_{q+1} \\ \hline \mathbb{1}_{q+1} & \mathbb{1}_{q+1}\end{array}\right]$,

2)

$$
\left[\begin{array}{c|c}
\mathbb{1}_{q+1} & 0 \\
\hline \mathbb{1}_{q} 0 & \mathbb{1}_{q}
\end{array}\right],\left[\begin{array}{c|c}
\mathbb{1}_{q} & \mathbb{1}_{q} 0 \\
\hline 0 & \mathbb{1}_{q+1}
\end{array}\right],\left[\begin{array}{l|l}
\mathbb{1}_{q+1} & \mathbb{1}_{q+1} \\
\hline J_{q+1} & \mathbb{1}_{q+1}
\end{array}\right],\left[\begin{array}{l|l}
\mathbb{1}_{q+1} & J_{q+1} \\
\hline \mathbb{1}_{q+1} & \mathbb{1}_{q+1}
\end{array}\right],
$$

3) $\left[\begin{array}{l|l}A(Q) & \mathbb{1}_{q+1} \\ \hline \mathbb{1}_{q+1} & \mathbb{1}_{q+1}\end{array}\right]$, 
where

$$
J_{q+1}=\left[\begin{array}{cccccc}
0 & 1 & 0 & \cdot & 0 & 0 \\
0 & 0 & 1 & \cdot & 0 & 0 \\
0 & 0 & 0 & \cdot & 0 & 0 \\
\cdot & \cdot & \cdot & \cdot & . & . \\
0 & 0 & 0 & \cdot & 0 & 1 \\
0 & 0 & 0 & \cdot & 0 & 0
\end{array}\right]
$$

and where $Q=P^{r}$ is a power of an irreducible unitary polynomial $P \neq X, X-1$ (see 2.5).

For this sake, we consider the following summands of $X_{w}$

$$
\begin{array}{ll}
E=\widehat{a_{1}} \oplus \widehat{a_{2}} \oplus \cdots \oplus \widehat{a_{r}} \oplus\{e\}, \quad E^{\prime}=\left\{e^{\wedge}\right\} \oplus \widehat{b_{r}} \oplus \cdots \widehat{b_{2}} \oplus \widehat{b_{1}}, \\
F=\widehat{c_{1}} \oplus \widehat{c_{2}} \oplus \cdots \oplus \widehat{c_{r}} \oplus\{f\}, \quad F^{\prime}=\left\{f^{\wedge}\right\} \oplus \widehat{d_{r}} \oplus \cdots \widehat{d_{2}} \oplus \widehat{d_{1}},
\end{array}
$$

and set $Z_{u}^{K}=E^{l} \oplus E^{\prime^{\prime}} \oplus F^{m^{\prime}} \oplus F^{\prime m}$.

The structure maps $\eta_{u i}^{K}: M_{i}^{-}\left(Z_{u}^{K}\right) \longrightarrow M_{i}^{+}\left(Z_{u}^{K}\right)$ are defined as sums

$$
\eta_{u i}^{K}=\eta_{u i E}^{K}+\eta_{u i E^{\prime}}^{K}+\eta_{u i F}^{K}+\eta_{u i F^{\prime}}^{K}+\nu_{u i}^{K},
$$

where the first four summands are induced by $f_{w i}: M_{i}^{-}\left(X_{w}\right) \longrightarrow M_{i}^{+}\left(X_{w}\right)$. For instance, $\eta_{u i E}^{K}$ is the composition

$$
M_{i}^{-}\left(Z_{u}^{K}\right) \stackrel{\mathrm{pr}}{\longrightarrow} M_{i}^{-}(E)^{l} \stackrel{\mathrm{im}}{\longrightarrow} M_{i}^{-}\left(X_{w}\right)^{l} \stackrel{f_{w i}^{l}}{\longrightarrow} M_{i}^{+}\left(X_{w}\right)^{l} \stackrel{\mathrm{pr}}{\longrightarrow} M_{i}^{+}(E)^{l} \stackrel{\mathrm{im}}{\longrightarrow} M_{i}^{+}\left(Z_{u}^{K}\right) .
$$

The last summand $\nu_{u i}^{K}$ is also a composition, namely,

$$
\begin{array}{r}
M_{i}^{-}\left(Z_{u}^{K}\right) \stackrel{\mathrm{pr}}{\longrightarrow} M_{i}^{-}(E)^{l} \oplus M_{i}^{-}\left(E^{\prime}\right)^{l^{\prime}} \stackrel{\mathrm{im}}{\longrightarrow} M_{i}^{-}\left(X_{w}\right)^{l} \oplus M_{i}^{-}\left(X_{w}\right)^{l^{\prime}} \\
g_{w i \pi+1}^{l} \stackrel{\oplus g_{w i}^{l^{\prime}}}{\longrightarrow}{ }^{2+2 r+2} k^{l} \oplus k^{l^{\prime}} \stackrel{K}{\longrightarrow} k^{m} \oplus k^{m^{\prime}} h_{w i \pi+2 r+2}^{m} \oplus h_{w i 2 \pi+1}^{m^{\prime}} \longrightarrow \\
M_{i}^{+}\left(X_{w}\right)^{m} \oplus M_{i}^{+}\left(X_{w}\right)^{m^{\prime}} \stackrel{\mathrm{pr}}{\longrightarrow} M_{i}^{+}\left(F^{\prime}\right)^{m} \oplus M_{i}^{+}(F)^{m^{\prime}} \stackrel{\mathrm{im}}{\longrightarrow} M_{i}^{+}\left(Z_{u}^{K}\right)
\end{array}
$$

if $a_{1} \in S_{i}^{-}$

$$
\begin{aligned}
& M_{i}^{+}\left(Z_{u}^{K}\right) \stackrel{\mathrm{im}}{\longleftarrow} M_{i}^{+}(E)^{l} \oplus M_{i}^{+}\left(E^{\prime}\right)^{l^{\prime}} \stackrel{\mathrm{pr}}{\longleftarrow} M_{i}^{+}\left(X_{w}\right)^{l} \oplus M_{i}^{+}\left(X_{w}\right)^{l^{\prime}} \\
& h_{w i \pi+1}^{l} \stackrel{\oplus h_{w i \pi+2 r+2}^{l^{\prime}}}{\longleftarrow} k^{l} \oplus k^{l^{\prime}} \stackrel{K^{\prime}}{\longleftarrow} k^{m} \oplus k^{m^{\prime}} g_{w i \pi+2 r+2}^{m} \oplus g_{w i 2 \pi+1}^{m^{\prime}} \\
& M_{i}^{-}\left(X_{w}\right)^{m} \oplus M_{i}^{-}\left(X_{w}\right)^{m^{\prime}} \stackrel{\mathrm{im}}{\longleftarrow} M_{i}^{-}\left(F^{\prime}\right)^{m} \oplus M_{i}^{-}(F)^{m^{\prime}} \stackrel{\mathrm{pr}}{\longleftarrow} M_{i}^{-}\left(Z_{u}^{K}\right)
\end{aligned}
$$

if $a_{1} \in S_{i}^{+}$, where $K^{\prime}=\left[\begin{array}{l|l}D & C \\ \hline B & A\end{array}\right]$ if $K$ is one of matrices listed in 1), and $K^{\prime}=K$ otherwise. The $\nu_{u i}^{K}$ is zero if $a_{1} \notin S_{i}^{-} \cup S_{i}^{+}$. 
As an example, we consider the bush of 2.3 and the case

$$
u_{0} u_{1} \cdots u_{\pi-1}=a e a^{\sim} b^{\sim} e^{\wedge} b .
$$

The structure maps are then visualized by the following diagram:
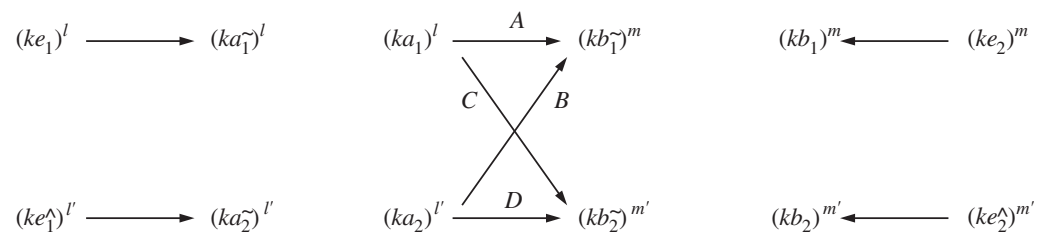

and the corresponding matrices are

$c$
$a^{\sim}\left[\begin{array}{cc|cc}0 & \mathbb{1}_{m} & 0 & 0 \\ 0 & 0 & 0 & \mathbb{1}_{m^{\prime}} \\ \hline \hline \mathbb{1}_{l} & 0 & 0 & 0 \\ 0 & 0 & \mathbb{1}_{l^{\prime}} & 0\end{array}\right]$

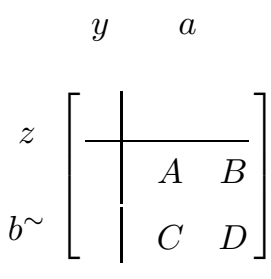

In view of our classification, we finally choose a set $\Omega_{4}$ of symmetric periodic catenations which contains one representative of each class $\coprod_{p \in \mathbb{Z}}[u\{p\}]$. For each $(u, K) \in \Omega_{4} \times \mathcal{Q}$ the preceding construction then provides a representation $R(u, K)$. A representation of $S$ isomorphic to such an $R(u, K)$ will be called a dimidiate band.

2.7. Main Theorem. Each indecomposable representation of a (completed) bush $S$ is a string (asymmetric or dimidiate) or a band (asymmetric or dimidiate). The represetations $R(\delta)$, where

$$
\delta \in \Omega_{1} \amalg \Omega_{2} \times\{0,1\} \amalg \Omega_{3} \times \mathcal{P} \amalg \Omega_{4} \times \mathcal{Q},
$$

are indecomposable and pairwise non-isomorphic.

The proof of the main theorem is based on the reduction in section 3 and will be given in section 4 .

2.8. Remark. (a) Let $w=w_{1} w_{2} \cdots w_{t}$ be an asymmetric catenation. then the reverse catenation $w^{*}=w_{t}^{*} \cdots w_{2}^{*} w_{1}^{*}$ is also asymmetric. By the construction of $R(w)$ and $R\left(w^{*}\right)$, we may identify $X_{w}$ with $X_{w^{*}}$ by identifying $\widehat{w_{i}}$ with $\widehat{w_{t-i+1}^{*}}$ 
for $1 \leq i \leq t$. In fact, one sees easily that such an identification induces an isomorphism between $R(w)$ and $R\left(w^{*}\right)$.

(b) Let $w=w_{1} w_{2} \cdots w_{t}$ be a symmetric catenation. Then $w$ is of the form

$$
w=a_{1} a_{2} \cdots a_{s} e b_{s} \cdots b_{2} b_{1},
$$

where $\left[a_{s}^{*} \cdots a_{2}^{*} a_{1}^{*}\right]=\left[b_{s} \cdots b_{2} b_{1}\right]$ and $e \neq e^{\wedge}$. Set

$$
v=a_{1} a_{2} \cdots a_{s} e a_{s}^{*} \cdots a_{2}^{*} a_{1}^{*} .
$$

Then $v$ is a symmetric catenation and equivalent to $w$. By the construction in 2.2 , one easily sees that $\left(X_{v} ; f_{v 1}, \cdots, f_{v n}\right)$ is decompose into a direct sum of two representations.

(c) Let $u=\left(u_{i}\right)_{i \in \mathbb{Z}}$ be an asymmetric periodic catenations of period $\pi$. Let $v=\left(v_{i}\right)_{\mathbb{Z}}$ be such that $v_{k \pi+i}=u_{i}$ for all $k \in \mathbb{Z}$ and $0 \leq i \leq \pi-1$. Then $v$ is also an asymmetric periodic catenation of period $\pi$ which is eqiuvalent to $u$. By the construction in 2.5, there holds that $R(u, Q) \cong R(v, Q)$ for each $Q \in \mathcal{P}$. Moreover, by changing basis vectors, one can easily prove that $R(v, Q) \cong R(v\{p\}, Q)$ for all $p \in \mathbb{Z}$. Thus $u$ and $v\{p\} \quad(p \in \mathbb{Z})$ provide the same family of isoclasses of representations of $S$.

Further, the reverse catenation $v^{*}$ of $v$ is asymmetric. By the construction in 2.5 , for each

$$
Q=P^{l}=X^{m l}-a_{1} X^{m l-1}-a_{2} X^{m l-2}-\cdots-a_{m l}, \quad l \geq 1
$$

in $\mathcal{P}$, we set $Q^{\prime}(X)=(-1) \frac{1}{a_{m l}} X^{m l} Q\left(\frac{1}{X}\right) \in \mathcal{P}$, then there holds that $R(v, Q) \cong$ $R\left(v^{*}, Q^{\prime}\right)$ since $A\left(Q^{\prime}\right)=A(Q)^{-1}$. Conversely, $R\left(v^{*}, Q\right) \cong R\left(v, Q^{\prime}\right)$. Thus $v$ and $v^{*}$ provide the same family of isoclasses of representations of $S$.

(d) Let $u=\left(u_{i}\right)_{i \in \mathbb{Z}}$ be a symmetric periodic catenations of period $\pi$. As in (c), let $v=\left(v_{i}\right)_{i \in \mathbb{Z}}$ be such that $v_{k \pi+i}=u_{i}$ for all $k \in \mathbb{Z}$ and $0 \leq i \leq \pi-1$. Then $v$ is also a symmetric periodic catenation of period $\pi$ which is eqiuvalent to $u$. By changing basis vectors, there holds that $R(u, K) \cong R(v\{p\}, K)$ for each $K \in \mathcal{Q}$ and each $p \in \mathbb{Z}$. Hence $u$ and $v\{p\}(p \in \mathbb{Z})$ provide the same family of isoclasses of representations of $S$.

\section{A reduction of representations of bushes}

In this section, we shall formulate the algorithm in [3] with respect to tangles formed by sequences of modules. We shall see in next section that such an algorithm will lead us to an efficient reduction of representations of bushes. All the proofs are analogous to those in [3]. We omit them.

3.1. Let $S=\left(S_{1}^{-}, \cdots, S_{n}^{-} ; S_{1}^{+}, \cdots S_{n}^{+} ; \sim\right)$ ba a bush as in 1.3 and $\left(M^{-}, M^{+}\right)$the tangle associated with $S$. For each representation $\left(X ; f_{1}, \cdots, f_{n}\right)$ of $\left(M^{-}, M^{+}\right)$, we denote by $f$ the sequence $\left(f_{1}, \cdots, f_{n}\right)$ and simply write $(X ; f)$ for $\left(X ; f_{1}, \cdots, f_{n}\right)$. 
Let $1 \leq i \leq n$. We start with submodules $K^{-} \subseteq L^{-}$of $M_{i}^{-}$and submodules $K^{+} \subseteq L^{+}$of $M_{i}^{+}$. We are interested in the representations $(X ; f)=$ $\left(X ; f_{1}, \cdots, f_{i}, \cdots f_{n}\right)$ of $\left(M^{-}, M^{+}\right)$which satisfy $f_{i}\left(K^{-}(X)\right) \subseteq K^{+}(X)$ and $f_{i}\left(L^{-}(X)\right) \subseteq L^{+}(X)$.

From now on, for each $X \in \mathcal{A}$, we fix subspaces $U_{1}^{-}(X), U_{2}^{-}(X)$ of $M_{i}^{-}(X)$ and subspaces $U_{1}^{+}(X), U_{2}^{+}(X)$ of $M_{i}^{+}(X)$ such that

$$
\begin{array}{ll}
L^{-}(X)=K^{-}(X) \oplus U_{1}^{-}(X), & M_{i}^{-}(X)=L^{-}(X) \oplus U_{2}^{-}(X) \\
L^{+}(X)=U_{1}^{+}(X) \oplus K^{+}(X), & M_{i}^{+}(X)=U_{2}^{+}(X) \oplus L^{+}(X) .
\end{array}
$$

Then for each representation $(X ; f)$ of $\left(M^{-}, M^{+}\right)$, the $f_{i}$ can be written as the form:

$$
\begin{aligned}
& f_{i}=\left[\begin{array}{lll}
f_{i 1} & f_{i 2} & f_{i 3} \\
f_{i 4} & f_{i 5} & f_{i 6} \\
f_{i 7} & f_{i 8} & f_{i 9}
\end{array}\right]: M_{i}^{-}(X)=K^{-}(X) \oplus U_{1}^{-}(X) \oplus U_{2}^{-}(X) \\
& \longrightarrow U_{2}^{+}(X) \oplus U_{1}^{+}(X) \oplus K^{+}(X)=M_{i}^{+}(X) .
\end{aligned}
$$

To the tangle $\left(M^{-}, M^{+}\right)$we now attach a new tangle as follows. First, we denote by $\mathcal{B}$ the full subcategory of $\operatorname{rep}\left(M^{-}, M^{+}\right)$formed by representations $(X ; \tilde{\rho})$, where $\rho: U_{1}^{-}(X) \rightarrow U_{1}^{+}(X)$ is a $k$-linar map and $\tilde{\rho}$ denote the sequence $\left(0, \cdots, 0, \rho_{0}, 0, \cdots, 0\right)$ with $\rho_{0}$ of the form:

$$
\begin{aligned}
& \rho_{0}=\left[\begin{array}{lll}
0 & 0 & 0 \\
0 & \rho & 0 \\
0 & 0 & 0
\end{array}\right]: M_{i}^{-}(X)=K^{-}(X) \oplus U_{1}^{-}(X) \oplus U_{2}^{-}(X) \\
& \longrightarrow U_{2}^{+}(X) \oplus U_{1}^{+}(X) \oplus K^{+}(X)=M_{i}^{+}(X) .
\end{aligned}
$$

Further, for each $(X ; \tilde{\rho}) \in \mathcal{B}$, we define

$$
\begin{aligned}
& N_{j}^{-}(X ; \tilde{\rho}):=M_{j}^{-}(X), N_{j}^{+}(X, \tilde{\rho}):=M_{j}^{+}(X), \text { for all } j \neq i, \\
& N_{i}^{-}(X ; \tilde{\rho}):=\operatorname{Ker} \tilde{\rho}=K^{-}(X) \oplus \operatorname{Ker} \rho \oplus U_{2}^{-}(X), \\
& \text { and } \quad N_{i}^{+}(X ; \tilde{\rho}):=\operatorname{Coker} \tilde{\rho}=U_{2}^{+}(X) \oplus \operatorname{Coker} \rho \oplus K^{+}(X) .
\end{aligned}
$$

For a morphism $\mu:(X ; \tilde{\rho}) \rightarrow\left(X^{\prime} ; \tilde{\rho}^{\prime}\right)$, we denote by $N_{j}^{-}(\mu)$ and $N_{j}^{+}(\mu)$ the $k$-linear maps induced respectively by $M_{j}^{-}(\mu)$ and $M_{j}^{+}(\mu)$ for $1 \leq j \leq n$. Then we obtain two sequences of modules $N^{-}=\left(N_{1}^{-}, \cdots, N_{n}^{-}\right)$and $N^{+}=\left(N_{1}^{+}, \cdots, N_{n}^{+}\right)$ over $\mathcal{B}$, that is, a tangle $\left(N^{-}, N^{+}\right)$over $\mathcal{B}$. Moreover, the modules $N_{i}^{-}$and $N_{i}^{+}$ admit respectively submodules $J^{-}$and $J^{+}$such that

$$
J^{-}(X, \tilde{\rho})=K^{-}(X) \oplus \operatorname{Ker} \rho, \quad J^{+}(X, \tilde{\rho})=K^{+}(X) .
$$


Finally, we denote by $\mathcal{M}$ the full subcategory of $\operatorname{rep}\left(M^{-}, M^{+}\right)$formed by representations $(X ; f)$ satisfying $f_{i}\left(K^{-}(X)\right) \subseteq K^{+}(X)$ and $f_{i}\left(L^{-}(X)\right) \subseteq L^{+}(X)$, and by $\mathcal{N}$ the full subcategory of $\operatorname{rep}\left(N^{-}, N^{+}\right)$formed by representations $((X, \tilde{\rho}) ; h)=$ $\left((X ; \tilde{\rho}) ; h_{1}, \cdots h_{n}\right)$ satisfying $h_{i}\left(J^{-}(X, \tilde{\rho})\right) \subseteq J^{+}(X, \tilde{\rho})$. Our purpose is to build up a relation between categories $\mathcal{M}$ and $\mathcal{N}$.

3.2. From now on, we suppose that $K^{-}=\mathcal{R}^{i_{1}} M_{i}^{-}$and $L^{-}=\mathcal{R}^{i_{2}} M_{i}^{-}$for some $i_{1} \geq i_{2}$, and that $K^{+}=\mathcal{R}^{j_{1}} M_{i}^{+}$and $L^{+}=\mathcal{R}^{j_{2}} M_{i}^{+}$for some $j_{1} \geq j_{2}$.

In order to establish a reduction from objects of $\mathcal{M}$ to those of $\mathcal{N}$, for each $(X ; \tilde{\rho})$ in $\mathcal{B}$, we choose a supplement $T^{-}(X ; \tilde{\rho})$ of $\operatorname{Ker} \rho$ in $U_{1}^{-}(X)$ and a supplement $T^{+}(X ; \tilde{\rho})$ of $\operatorname{Im} \rho$ in $U_{1}^{+}(X)$. Then $\rho_{0}$ can be written in the form:

$$
\begin{aligned}
\rho_{0}= & {\left[\begin{array}{cccc}
0 & 0 & 0 & 0 \\
0 & 0 & \bar{\rho} & 0 \\
0 & 0 & 0 & 0 \\
0 & 0 & 0 & 0
\end{array}\right]: M_{i}^{-}(X)=K^{-}(X) \oplus \operatorname{Ker} \rho \oplus T^{-}(X ; \tilde{\rho}) \oplus U_{2}^{-}(X) } \\
& \longrightarrow U_{2}^{+}(X) \oplus \operatorname{Im} \rho \oplus T^{+}(X ; \tilde{\rho}) \oplus K^{+}(X)=M_{i}^{+}(X),
\end{aligned}
$$

where $\bar{\rho}: T^{-}(X ; \tilde{\rho}) \rightarrow \operatorname{Im} \rho$ is induced by $\rho$.

Further, for each object $(X ; f)$ in $\mathcal{M}$, the $f_{i}$ is of the form:

$$
\begin{gathered}
f_{i}=\left[\begin{array}{ccc}
0 & 0 & f_{i 1} \\
0 & f_{i 2} & f_{i 3} \\
f_{i 4} & f_{i 5} & f_{i 6}
\end{array}\right]: M_{i}^{-}(X)=K^{-}(X) \oplus U_{1}^{-}(X) \oplus U_{2}^{-}(X) \\
\longrightarrow U_{2}^{+}(X) \oplus U_{1}^{+}(X) \oplus K^{+}(X)=M_{i}^{+}(X) .
\end{gathered}
$$

In such a way, $(X ; f)$ gives rise to an object $\left(X ; \tilde{f_{i 2}}\right)$ in $\mathcal{B}$. By further decomposing $U_{1}^{-}(X)$ and $U_{1}^{+}(X)$, we infer that $f_{i}$ has the form:

$$
\begin{aligned}
f_{i}=\left[\begin{array}{cccc}
0 & 0 & 0 & f_{i 1} \\
0 & 0 & \tilde{f_{i 2}} & f_{i 3}^{\prime \prime} \\
0 & 0 & 0 & f_{i 3}^{\prime} \\
f_{i 4} & f_{i 5}^{\prime} & f_{i 5}^{\prime \prime} & f_{i 6}
\end{array}\right]: M_{i}^{-}(X)=K^{-}(X) \oplus \operatorname{Ker} f_{2} \oplus T^{-}\left(X ; \tilde{f_{i 2}}\right) \oplus U_{2}^{-}(X) \\
\longrightarrow U_{2}^{+}(X) \oplus \operatorname{Im} f_{2} \oplus T^{+}\left(X ; \tilde{f_{i 2}}\right) \oplus K^{+}(X)=M_{i}^{+}(X) .
\end{aligned}
$$

Since the tangle $\left(M^{-}, M^{+}\right)$is rodded, $(X ; f)$ is isomorphic to the object $\left(X ; f^{\prime}\right)=\left(X ; f_{1}, \cdots, f_{i-1}, f_{i}^{\prime}, f_{i+1}, \cdots, f_{n}\right)$ with $f_{i}^{\prime}$ of the form:

$$
\begin{array}{r}
f_{i}^{\prime}=\left[\begin{array}{cccc}
0 & 0 & 0 & f_{i 1} \\
0 & 0 & \tilde{f_{i 2}} & 0 \\
0 & 0 & 0 & f_{i 3}^{\prime} \\
f_{i 4} & f_{i 5}^{\prime} & 0 & f_{i 6}^{\prime}
\end{array}\right]: M_{i}^{-}(X)=K^{-}(X) \oplus \operatorname{Ker} f_{2} \oplus T^{-}\left(X ; \tilde{f_{i 2}}\right) \oplus U_{2}^{-}(X) \\
\longrightarrow U_{2}^{+}(X) \oplus \operatorname{Im} f_{2} \oplus T^{+}\left(X ; \tilde{f_{i 2}}\right) \oplus K^{+}(X)=M_{i}^{+}(X)
\end{array}
$$


where $f_{i 6}^{\prime}=f_{i 6}-f_{i 5}^{\prime \prime}{\overline{f_{i 2}}}^{-1} f_{i 3}^{\prime \prime}$. Finally, we denote by $\eta_{(X ; f)}$ an isomorphism from $(X ; f)$ to $\left(X ; f^{\prime}\right)$.

Thus each object $(X ; f)$ then gives rise to an object $\left(\left(X ; \tilde{f_{i 2}}\right), \hat{f}\right)$ in $\mathcal{N}$ with $\hat{f}=\left(f_{1}, \cdots, f_{i-1}, \hat{f}_{i}, f_{i+1}, \cdots, f_{n}\right)$, where $\hat{f}_{i}$ is of the form

$$
\begin{array}{r}
\hat{f}_{i}=\left[\begin{array}{ccc}
0 & 0 & f_{i 1} \\
0 & 0 & p_{\left(X ; \tilde{f_{i 2}}\right)} \\
f_{i 4} & f_{i 5}^{\prime} & f_{i 6}^{\prime}
\end{array}\right]: N_{i}^{-}\left(X ; \tilde{f_{i 2}}\right)=K^{-}(X) \oplus \operatorname{Ker} f_{2} \oplus U_{2}^{-}(X) \\
\longrightarrow U_{2}^{+}(X) \oplus \operatorname{Coker} f_{2} \oplus K^{+}(X)=N_{i}^{+}\left(X ; \tilde{f_{i 2}}\right) .
\end{array}
$$

(Here $p_{\left(X ; \tilde{\left.f_{i 2}\right)}\right.}$ denotes the restriction of the canonical projection $\pi_{\left(X ; \tilde{f_{i 2}}\right)}: U_{1}^{+}(X)$ $\rightarrow \operatorname{Coker} f_{2}=U_{1}^{+}(X) / \operatorname{Im} f_{2}$ to $T^{+}\left(X ; \tilde{f_{i 2}}\right)$.)

3.3. Remark. Up to isomorphisms, the representation $\left(\left(\underset{\tilde{f}}{f_{i 2}}\right), \hat{f}\right)$ induced by $(X ; f)$ is independent on the choice of supplements $T^{-}\left(X ; \tilde{f_{i 2}}\right)$ and $T^{+}\left(X ; \tilde{f_{i 2}}\right)$.

3.4. In view of Remark 3.3 , for each $(X ; \tilde{\rho})$ in $\mathcal{B}$, we may fix a supplement $T^{-}(X ; \tilde{\rho})$ of $\operatorname{Ker} \rho$ in $U_{1}^{-}(X)$ and a supplement $T^{+}(X ; \tilde{\rho})$ of $\operatorname{Im} \rho$ in $U_{1}^{+}(X)$. By the discussion in 3.2 , each object $(X ; f)$ in $\mathcal{M}$ then gives rise uniquely to an object $\left(\left(X ; \tilde{f_{i 2}}\right) ; \hat{f}\right)$ in $\mathcal{N}$.

Let $(X ; f)$ and $(Y ; g)$ be objects in $\mathcal{M}$ and $\mu$ a morphism from $(X ; f)$ to $(Y ; g)$. With $\mu$ we now associate a morphism from $\left(\left(X ; \tilde{f_{i 2}}\right) ; \hat{f}\right)$ to $\left(\left(Y ; \tilde{g_{i 2}}\right) ; \hat{g}\right)$.

Again by 3.2 , one has that $\tilde{\mu}=: \eta_{(Y ; g)} \mu\left(\eta_{(X ; f)}\right)^{-1}$ is a morphism from $\left(X ; f^{\prime}\right)$ $=\left(X ; f_{1}, \cdots, f_{i}^{\prime}, \cdots, f_{n}\right)$ to $\left(Y ; g^{\prime}\right)=\left(Y ; g_{1}, \cdots, g_{i}^{\prime}, \cdots, g_{n}\right)$, where $f_{i}^{\prime}$ and $g_{i}^{\prime}$ are of the forms:

$$
\begin{array}{r}
f_{i}^{\prime}=\left[\begin{array}{cccc}
0 & 0 & 0 & f_{i 1} \\
0 & 0 & \overline{f_{i 2}} & 0 \\
0 & 0 & 0 & f_{i 3}^{\prime} \\
f_{i 4} & f_{i 5}^{\prime} & 0 & f_{i 6}^{\prime}
\end{array}\right]: M_{i}^{-}(X)=K^{-}(X) \oplus \operatorname{Ker} f_{2} \oplus T^{-}\left(X ; \tilde{f_{i 2}}\right) \oplus U_{2}^{-}(X) \\
\longrightarrow U_{2}^{+}(X) \oplus \operatorname{Im} f_{2} \oplus T^{+}\left(X ; \tilde{f_{i 2}}\right) \oplus K^{+}(X)=M_{i}^{+}(X)
\end{array}
$$

and

$$
\begin{array}{r}
g^{\prime}=\left[\begin{array}{cccc}
0 & 0 & 0 & g_{i 1} \\
0 & 0 & g_{i 2} & 0 \\
0 & 0 & 0 & g_{i 3}^{\prime} \\
g_{i 4} & g_{i 5} & 0 & g_{i 6}^{\prime}
\end{array}\right]: M_{i}^{-}(Y)=K^{-}(Y) \oplus \operatorname{Ker} g_{2} \oplus T^{-}\left(Y ; \tilde{g_{i 2}}\right) \oplus S_{2}^{-}(Y) \\
\longrightarrow S_{2}^{+}(Y) \oplus \operatorname{Im} g_{2} \oplus T^{+}\left(Y ; \tilde{g_{i 2}}\right) \oplus K^{+}(Y)=M_{i}^{+}(Y)
\end{array}
$$


Further, the maps $M_{i}^{-}(\tilde{\mu})$ and $M_{i}^{+}(\tilde{\mu})$ can be written as the following forms:

$$
\begin{aligned}
M_{i}^{-}(\tilde{\mu})=\left[\begin{array}{cccc}
a_{11} & a_{12} & a_{13} & a_{14} \\
0 & a_{22} & a_{23} & a_{24} \\
0 & a_{32} & a_{33} & a_{34} \\
0 & 0 & 0 & a_{44}
\end{array}\right]: M_{i}^{-}(X) & \\
& =K^{-}(X) \oplus \operatorname{Ker}_{2} \oplus T^{-}\left(X ; \tilde{f_{i 2}}\right) \oplus U_{2}^{-}(X) \\
& \longrightarrow K^{-}(Y) \oplus \operatorname{Ker}_{2} \oplus T^{-}\left(Y ; \tilde{g_{i 2}}\right) \oplus S_{2}^{-}(Y)=M_{i}^{-}(Y)
\end{aligned}
$$

and

$$
\begin{aligned}
M_{i}^{+}(\tilde{\mu})=\left[\begin{array}{cccc}
b_{11} & 0 & 0 & 0 \\
b_{21} & b_{22} & b_{23} & 0 \\
b_{31} & b_{32} & b_{33} & 0 \\
b_{41} & b_{42} & b_{43} & b_{44}
\end{array}\right]: & M_{i}^{+}(X) \\
& \\
& =U_{2}^{+}(X) \oplus \operatorname{Im} f_{2} \oplus T^{+}\left(X ; \tilde{f_{i 2}}\right) \oplus K^{+}(X) \\
& \longrightarrow S_{2}^{+}(Y) \oplus \operatorname{Im} g_{2} \oplus T^{+}\left(Y ; \tilde{g_{i 2}}\right) \oplus K^{+}(Y)=M_{i}^{+}(Y) .
\end{aligned}
$$

Then there holds that

$$
\begin{aligned}
& {\left[\begin{array}{cccc}
0 & 0 & 0 & g_{i 1} \\
0 & 0 & g_{i 2} & 0 \\
0 & 0 & 0 & g_{i 3}^{\prime} \\
g_{i 4}^{\prime} & g_{i 5}^{\prime} & 0 & g_{i 6}^{\prime}
\end{array}\right]\left[\begin{array}{cccc}
a_{11} & a_{12} & a_{13} & a_{14} \\
0 & a_{22} & a_{23} & a_{24} \\
0 & a_{32} & a_{33} & a_{34} \\
0 & 0 & 0 & a_{44}
\end{array}\right]} \\
& =\left[\begin{array}{cccc}
b_{11} & 0 & 0 & 0 \\
b_{21} & b_{22} & b_{23} & 0 \\
b_{31} & b_{32} & b_{33} & 0 \\
b_{41} & b_{42} & b_{43} & b_{44}
\end{array}\right]\left[\begin{array}{cccc}
0 & 0 & 0 & f_{i 1} \\
0 & 0 & \overline{f_{i 2}} & 0 \\
0 & 0 & 0 & f_{i 3}^{\prime} \\
f_{i 4} & f_{i 5}^{\prime} & 0 & f_{i 6}^{\prime}
\end{array}\right]
\end{aligned}
$$

since $\tilde{\mu}$ is a morphism.

It then follows that $a_{32}=0$ and $b_{32}=0$. Since $\left(M^{-}, M^{+}\right)$is rodded, there is a morphism $\mu^{\prime} \in \mathcal{R}_{\mathcal{A}}(X, Y)$ such that

$$
\begin{aligned}
& \left(M_{j}^{-}\left(\mu^{\prime}\right), M_{j}^{+}\left(\mu^{\prime}\right)=(0,0) \text { for all } j \neq i\right. \\
& \left(M_{i}^{-}\left(\mu^{\prime}\right), M_{i}^{+}\left(\mu^{\prime}\right)\right)=\left(\left[\begin{array}{cccc}
0 & 0 & 0 & 0 \\
0 & 0 & 0 & 0 \\
0 & 0 & 0 & a_{34} \\
0 & 0 & 0 & 0
\end{array}\right],\left[\begin{array}{cccc}
0 & 0 & 0 & 0 \\
0 & 0 & 0 & 0 \\
0 & 0 & 0 & 0 \\
0 & b_{42} & 0 & 0
\end{array}\right]\right) .
\end{aligned}
$$

We then set $\hat{\mu}=\tilde{\mu}-\mu^{\prime}: X \rightarrow Y$. It is easy to show that $\hat{\mu}$ is a morphism from $\left(X ; \tilde{f_{i 2}}\right)$ to $\left(Y ; \tilde{g_{i 2}}\right)$. By $(1)$ there also holds that

$$
\hat{g} N_{i}^{-}(\hat{\mu})=N_{i}^{+}(\hat{\mu}) \hat{f}
$$


that is, $\hat{\mu}$ is a morphism from $\left(\left(X ; \tilde{f_{i 2}}\right) ; \hat{f}\right)$ to $\left(\left(Y ; \tilde{g_{i 2}}\right), \hat{g}\right)$.

As a conclusion, we obtain two correspondences $(X ; f) \longmapsto\left(\left(X ; \tilde{f_{i 2}}\right) ; \hat{f}\right)$ and $\mu \longmapsto \hat{\mu}$ which induce a functor

$$
\Phi: \mathcal{M} \longrightarrow \mathcal{N} / I
$$

such that $\Phi(X ; f)=\left(\left(X ; \tilde{f_{i 2}}\right) ; \hat{f}\right)$ and $\Phi(\mu)=\hat{\mu}+I$, where $I$ denotes the ideal of $\mathcal{N}$ generated by $\hat{\nu \mu}-\hat{\nu} \hat{\mu}$ for $\mu:(X ; f) \rightarrow(Y ; g)$ and $\nu:(Y ; g) \rightarrow(Z ; h)$ in $\mathcal{M}$.

Proposition. (1) The ideal I lies in the radical of $\mathcal{N}$.

(2) The functor $\Phi$ is an epivalence, i.e. $\Phi$ is full, hits each isoclass, and detects isomorphisms.

3.5. For the practical application, in certain situations it imports us to translate the reduction into the language of matrix problems. We illustrate the translation with an example: Let $S$ be the (non-complete) bush in 2.3, i.e. $S$ is formed by the following pairs of rods:

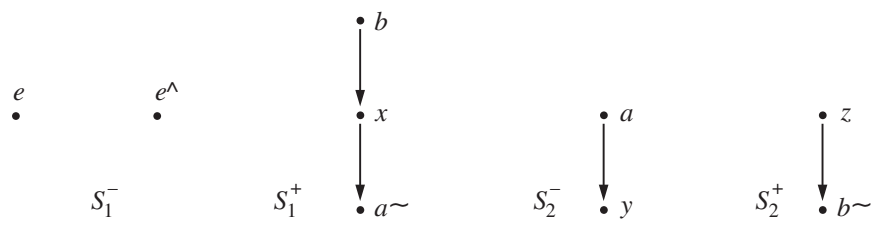

The associated tangle consists of two pairs of modules $\left(M_{1}^{-}, M_{1}^{+}\right)$and $\left(M_{2}^{-}, M_{2}^{+}\right)$. Let $\left(X ; f_{1}, f_{2}\right)$ be a representation of $S$. If $X$ is fixed, the chosen bases of $M_{i}^{-}(X)$ and of $M_{i}^{+}(X)$ provide us a matrix problem given by a pair of partitioned matrices

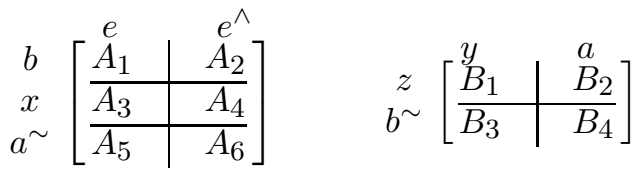

together with the following admissible transformations

(a) arbitrary row transformations within stripes $x$ and $z$ and abitrary column transformations within stripes $e, e^{\wedge}$ and $y$;

(b) row transformations within stripe $a^{\sim}$ coupled with the conjugate column transformations within stripe $a$, row transformations within stripe $b$ coupled with the same row transformations within stripe $b^{\sim}$ (Note that the number of rows in stripe $a^{\sim}$ equals to the number of columns in stripe $a$ and that the number of rows in stripe $b$ equals to the number of rows in stripe $b^{\sim}$ );

(c) additions of multiples of rows between different stripes are allowed from $b$ to $x$ and $a^{\sim}$, from $x$ to $a^{\sim}$, and from $z$ to $b^{\sim}$, additions of multiples of columns between different stripes are only allowed from $y$ to $a$. 
Thanks to the algorithm, we first reduce $\left[A_{1} \mid A_{2}\right]$ to the following form:

$$
\left[\begin{array}{ccccc|ccccc}
0 & \mathrm{I} & 0 & \mathrm{I} & 0 & 0 & \mathrm{I} & 0 & \mathrm{I} & 0 \\
\hdashline 0 & \mathrm{I} & 0 & \mathrm{I} & 0 & 0 & \mathrm{I} & \mathbb{1} & \mathrm{I} & 0 \\
\hdashline 0 & \mathrm{I} & \mathbb{1 1} & \mathrm{I} & 0 & 0 & \mathrm{I} & 0 & \mathrm{I} & 0 \\
\hdashline 0 & \mathrm{I} & 0 & \mathrm{I} & \mathbb{1} & 0 & \mathrm{I} & 0 & \mathrm{I} & \mathbb{1 1}
\end{array}\right]
$$

By performing admissible transformations, we reduce $A_{3}, A_{4}, A_{5}$ and $A_{6}$ to the following forms (the row partition of stripe $b$ induces a partition of stripe $b^{\sim}$ ):

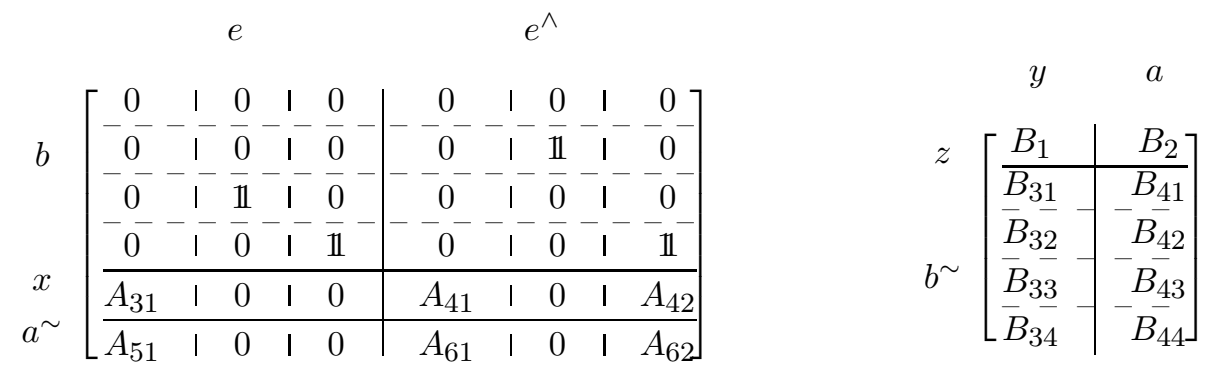

Thus we are reduced to the matrix problems described by the following matrices:

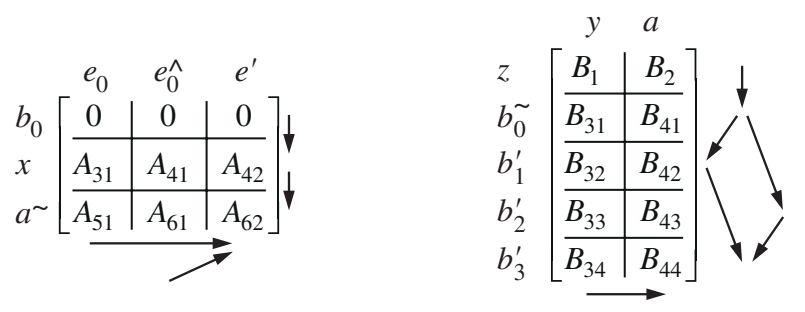

Without spoiling the reduced form of $\left[A_{1} \mid A_{2}\right]$, we can perform the following transformations to the matrices above:

(a') arbitrary row transformations within stripes $x, z, b_{0}^{\sim}, b_{1}^{\prime}$, and $b_{2}^{\prime}$, and abitrary column transformations within stripes $e_{0}, e_{0}^{\wedge}$, and $y$;

(b') row transformations within stripe $a^{\sim}$ (resp. $b_{2}$ ) coupled with the conjugate column transformations within stripe $a$ (resp. $\left.e^{\prime}\right)$,

(c') additions of multiples of rows and columns between different stripes are illustrated by the arrows in the figure above.

Thus the reduced matrices can be viewed as a matrix representation of a new bush $T$ given by the following pair of rods: 

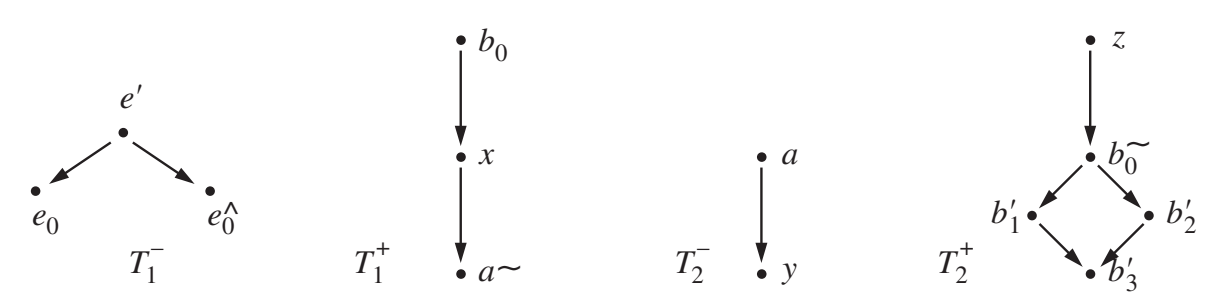

together with the equivalence relation such that $a \sim a^{\sim}, b_{0} \sim b_{0}^{\sim}$ and $e^{\prime} \sim b_{3}^{\prime}$. This matrix problem coincides with that obtained from the algorithm. For further reduction, one can reduce matrix $\left[A_{31} \mid A_{41}\right]$, and so on.

\section{The proof of the main theorem}

4.1. In this section, we shall keep all the notations in the preceding Sections. Let $S$ be a bush and $\left(X ; f_{1}, \cdots, f_{n}\right)$ a representation of $S$. By definition, the dimension of $\left(X ; f_{1}, \cdots, f_{n}\right)$ is

$$
\sum_{i=1}^{n}\left(\operatorname{dim}_{k} M_{i}^{-}(X)+\operatorname{dim}_{k} M_{i}^{+}(X)\right)
$$

where $M_{i}^{-}$and $M_{i}^{+}$are the modules associated with $S$ (see 1.3).

Let us now return and stick to complete bushes. We start from a complete bush $S=\left(S_{1}^{-}, \cdots, S_{n}^{-} ; S_{1}^{+}, \cdots, S_{n}^{+} ; \sim\right)$.

By abuse of notations in Section 2 , we call a representation $\left(X ; f_{1}, \cdots, f_{n}\right)$ of $S$ an asymmetric string if it is isomorphic to $R(w)$ for some asymmetric catenation $w$, a dimidiate string if it is isomorphic to a non-trivial summand of $\left(X_{v} ; f_{v 1}, \cdots, f_{v n}\right)$ for some symmetric catenation $v$, and an asymmetric (resp. a dimidiate) band if it is isomorphic to $R(u, Q)$ (resp. $R(u, K)$ ) for some asymmetric (resp. symmetric) periodic catenation $u$ and some $Q \in \mathcal{P}$ (resp. $K \in \mathcal{Q}$ ).

Let $(X ; f)=\left(X ; f_{1}, \cdots, f_{n}\right)$ be an indecomposable representation of $S$ with dimension $d$. Our objective is to prove by induction on $d$ that $\left(X ; f_{1}, \cdots, f_{n}\right)$ is a string or a band.

If $d=1$, it is clear that $\left(X ; f_{1}, \cdots, f_{n}\right)$ is a dimidiate string. We now suppose that $d>1$ and that every indecomposable representation of an arbitrary complete bush $T$ with dimension $<d$ is a string or a band.

If all $f_{i}$ vanish, $X$ is indecomposable in $\mathcal{A}$ and $\left(X ; f_{1}, \cdots, f_{n}\right)=(X ; 0, \cdots, 0)$ is an asymmetric string (since $d>1$ ). Otherwise, let $1 \leq i \leq n$ be such that $f_{i} \neq 0$. Then there are $m^{-}, m^{+} \in \mathbb{N}$ such that

i) $f_{i}\left(\mathcal{R}^{m^{-}+1} M_{i}^{-}(X)\right) \subseteq \mathcal{R}^{m^{+}+1} M_{i}^{+}(X)$,

ii) $f_{i}\left(\mathcal{R}^{m^{-}} M_{i}^{-}(X)\right) \subseteq \mathcal{R}^{m^{+}} M_{i}^{+}(X)$,

iii) the induced map

$$
\bar{f}_{i}: \mathcal{R}^{m^{-}} M_{i}^{-}(X) / \mathcal{R}^{m^{-}+1} M_{i}^{-}(X) \longrightarrow \mathcal{R}^{m^{+}} M_{i}^{+}(X) / \mathcal{R}^{m^{+}+1} M_{i}^{+}(X)
$$


is not zero, where $\mathcal{R}$ denotes the radical of $\mathcal{A}$.

We then set $K^{-}=\mathcal{R}^{m^{-}+1} M_{i}^{-}, L^{-}=\mathcal{R}^{m^{-}} M_{i}^{-}, K^{+}=\mathcal{R}^{m^{+}+1} M_{i}^{+}$, and $L^{+}=\mathcal{R}^{m^{+}} M_{i}^{+}$.

By Proposition 3.4 , we may reduce $(X ; f)$ to a representation $\left(\left(X ; \tilde{f_{i 2}}\right) ; \hat{f}\right)$ of a new tangle $\left(N^{-}, N^{+}\right)$over the aggregate $\mathcal{B}$ such that $\left(\left(X ; \tilde{f_{i 2}}\right) ; \hat{f}\right)$ is indecomposable and has dimension strictly less than $d$.

Since the lattices of submodules of $M_{i}^{-}$and $M_{i}^{+}$are rods, both the supports of $L^{-} / K^{-}$and of $L^{+} / K^{+}$contain one or two elements in $\mathcal{S}$. We examine the various cases seperately.

4.2. Case I. $\operatorname{supp}\left(L^{-} / K^{-}\right)=\{\bar{x}\}, \operatorname{supp}\left(L^{+} / K^{+}\right)=\{\bar{y}\}$ and $\bar{x} \neq \bar{y}$ for some $x \in S_{i}^{-}$and $y \in S_{i}^{+}$.

By way of example, we may suppose that $x^{\sim} \in S_{j_{1}}^{-}, y^{\sim} \in S_{j_{2}}^{-}$for some $j_{1} \neq$ $i, j_{2} \neq i$. All the other situations can be treated similarly.

In order to apply the algorithm described in 3.2-3.4, we choose the supplements $U_{1}^{-}, U_{2}^{-}, U_{1}^{+}$and $U_{2}^{+}$in the following canonical way: For each $a \in \mathcal{S}$, we set

$$
U_{1}^{-}(a)=\left\{\begin{array}{ll}
0 & \text { if } a \neq \bar{x} \\
k x & \text { if } a=\bar{x}
\end{array} \quad U_{2}^{-}(a)=\oplus_{u \in a, u \in P^{-}, u<x} k u\right.
$$

and

$$
U_{1}^{+}(a)=\left\{\begin{array}{ll}
0 & \text { if } a \neq \bar{y} \\
k y & \text { if } a=\bar{y}
\end{array} \quad U_{2}^{+}(a)=\oplus_{v \in a, v \in P^{+}, v<y} k v\right.
$$

where $\bar{x}$ and $\bar{y}$ denote the equivalence classes of $x$ and $y$ in $S$, respectively. Finally, for each $X \stackrel{\mu}{\cong} \oplus_{a \in \mathcal{S}} a^{n(a)} \in \mathcal{A}$, we set

$$
U_{i}^{-}(X)=M_{i}^{-}(\mu)^{-1}\left(\oplus_{a \in \mathcal{S}} U_{i}^{-}(a)^{n(a)}\right)
$$

and

$$
i=1,2 \text {. }
$$

$$
U_{i}^{+}(X)=M_{i}^{+}(\mu)^{-1}\left(\oplus_{a \in \mathcal{S}} U_{i}^{+}(a)^{n(a)}\right)
$$

The representations $(a ; 0)=(a ; 0, \cdots, 0), a \in \mathcal{S}$, and $(\bar{x} \oplus \bar{y} ; \eta)$, furnish a complete list of indecomposables in the aggregate $\mathcal{B}$, where $\eta$ denotes the sequence $\left(0, \cdots, 0, \eta_{i}=1,0, \cdots, 0\right)$. Then there holds that

$$
\begin{aligned}
& N_{j_{1}}^{-}(Y ; g)= \begin{cases}M_{j_{1}}^{-}(Y) & \text { if }(Y ; g)=(a ; 0) \\
k x^{\sim} & \text { if }(Y ; g)=(\bar{x} \oplus \bar{y}, \eta)\end{cases} \\
& N_{j_{2}}^{-}(X ; f)= \begin{cases}M_{j_{2}}^{-}(Y) & \text { if }(Y ; g)=(a ; 0) \\
k y^{\sim} & \text { if }(Y ; g)=(\bar{x} \oplus \bar{y}, \eta)\end{cases}
\end{aligned}
$$

We denote by $\overline{\mathcal{S}}$ the spectroid of $\mathcal{B}$ formed by representations $(a ; 0)=(a ; 0, \cdots, 0)$, $a \in \mathcal{S}$ and $(\bar{x} \oplus \bar{y} ; \eta)$. 
By $T=\left(T_{1}^{-}, \cdots, T_{n}^{-} ; T_{1}^{+}, \cdots, T_{n}^{+} ; \sim\right)$ we denote the bush formed by rods $T_{j_{1}}^{-}=S_{j_{1}}^{-} \amalg\left\{x_{1}\right\}, T_{j_{2}}^{-}=S_{j_{2}}^{-} \amalg\left\{y_{1}\right\}$, and $T_{l}^{\varepsilon}=S_{l}^{\varepsilon}$ for $(l, \varepsilon) \neq\left(j_{1},-\right),\left(j_{2},-\right)$. The union $\cup_{i=1}^{n}\left(T_{i}^{-} \cup T_{i}^{+}\right)$is equipped with the smallest order relation which contains that of $S$ induced by $S_{k}^{\varepsilon}$ and is such that $x^{\sim}>x_{1}, y^{\sim}<y_{1}$ and $z \gtrless x_{1}$ (resp. $\left.z \gtrless y_{1}\right)$ iff $z \gtrless x^{\sim}$ (resp. $z \gtrless y^{\sim}$ ). Finally we equip $T$ with the equivalence relation induced by $S$ and extended by $x_{1} \sim y_{1}$. The spectroid associated with $T$ (see 1.3) is denoted by $\mathcal{T}$.

An easy observation shows that the correspondence

$$
(\bar{z} ; 0) \longmapsto \bar{z}, \quad z \in S, \quad(\bar{x} \oplus \bar{y} ; \eta) \longmapsto \bar{x}_{1}=\bar{y}_{1}
$$

gives rise to an isomorphism from $\overline{\mathcal{S}}$ to $\mathcal{T}$. Therefore, by identifying $\overline{\mathcal{S}}$ with $\mathcal{T}$, the reduced form $\left(\left(X ; \tilde{f_{i 2}}\right), \hat{f}\right)$ of $(X ; f)$ can be considered as a representation of the new bush $T$.

By induction hypothesis, $\left(\left(X ; \tilde{f_{i 2}}\right) ; \hat{f}\right)$ is a string or a band which is associated with a catenation $v$ (finite or periodic) of $T$.

We denote by $w$ the catenation of $S$ obtained from $v$ by replacing each term $x_{1}$ by $x^{\sim} y$ and $y_{1}$ by $y^{\sim} x$.

We first consider the case where $v$ is an asymmetric catenation. Then $w$ is also an asymmetric catenation and $R(w)=\left(X_{w} ; f_{w 1}, \cdots, f_{w n}\right)$ is an asymmetric string. By the construction of $R(w)$, one sees that each part $x^{\sim} y$ or $y^{\sim} x$ in $w$ provides a summand $(\bar{x} \oplus \bar{y} ; \eta)$ of $\left(X_{w} ; \tilde{f}_{w i 2}\right)$. Thus $\left(X_{w} ; \tilde{f}_{w i 2}\right)$ and $X_{v}$ considered as objects in $\mathcal{B}$ are isomorphic. By identifying $\left(X_{w} ; \tilde{f}_{w i 2}\right)$ with $X_{v}$, the action of $\hat{f}_{w i}$ coincides with that of $f_{v i}$, so the representation $\left(\left(X_{w} ; \tilde{f}_{w i 2}\right) ; \hat{f}_{w}\right)$ is isomorphic to $R(v)$. By Proposition 3.4, we infer that $(X ; f) \cong R(w)$, that is, $(X, f)$ is an asymmetric string (The decisive point is the following: If a term $w_{r}$ of $w$ arises from some term $v_{q} \neq v_{q}^{\wedge}$, then $\left[w_{r-1}^{*} w_{r-2}^{*} \cdots\right] \leq\left[w_{r+1} w_{r+2} \cdots\right]$ is equivalent to $\left.\left[v_{q-1}^{*} v_{q-2}^{*} \cdots\right] \leq\left[v_{q+1} v_{q+2} \cdots\right]\right)$.

If $v$ is a symmetric catenation, so is $w$. One then obtains that $(X ; f)$ is isomorphic to a non-trivial summand of $\left(X_{w} ; f_{w 1}, \cdots, f_{w n}\right)$ since $\left.\left(X ; \tilde{f_{i 2}}\right) ; \hat{f}\right)$ is isomorphic to a non-trivial summand of $\left(X_{v} ; f_{v 1}, \cdots, f_{v n}\right)$, that is, $(X ; f)$ is a dimidiate string.

In the case where $v$ is a periodic catenation, one can similarly prove that $(X ; f) \cong R(w, Q) \quad($ resp. $R(w, K))$ according as $\left.\left(X ; \tilde{f_{i 2}}\right) ; \hat{f}\right) \cong R(v, Q)$ (resp. $R(v, K))$ for some $Q \in \mathcal{P}$ (resp. $K \in \mathcal{P})$, that is, an asymmetric (resp. a dimidiate) band.

4.3. Case II. $\operatorname{supp}\left(L^{-} / K^{-}\right)=\operatorname{supp}\left(L^{+} / K^{+}\right)=\{\bar{x}\}$ for some $x \in S_{i}^{-}$with $x^{\sim} \in S_{i}^{+}$.

In this case, one can easily see that the representations $(a ; 0), a \in \mathcal{S}$ and $\left((\bar{x})^{t} ; \eta(t)\right), t>1$, furnish a complete list of indecomposables in $\mathcal{B}$ which are not annihilated by $\mathcal{J}$, where $\mathcal{J}$ denotes the intersection of annihilators of all $N_{j}^{-}$and $N_{j}^{+}$, and where $\eta(t)$ denotes the sequence $\left(0, \cdots, 0, \eta(t)_{i}, 0, \cdots, 0\right)$ with $\eta(t)_{i}$ of 
the form:

$$
\eta(t)_{i}=\left[\begin{array}{ccccc}
0 & 1 & 0 & \ldots & 0 \\
0 & 0 & 1 & \ldots & 0 \\
\vdots & \vdots & \vdots & \ddots & \vdots \\
0 & 0 & 0 & \cdots & 1 \\
0 & 0 & 0 & \cdots & 0
\end{array}\right]: M_{i}^{-}\left((\bar{x})^{t}\right)=(k x)^{t} \longrightarrow\left(k x^{\sim}\right)^{t}=M_{i}^{+}\left((\bar{x})^{t}\right)
$$

(Note that the supplements $S_{1}^{-}, S_{2}^{-}, S_{1}^{+}$and $S_{2}^{+}$are chosen in a cononical way similar to the case I.)

Since $(X ; f)$ is finite dimensional, there exists an $L>0$ such that the induced representations $\left(X ; \tilde{f_{i 2}}\right)$ does not contain a summand isomorphic to some $\left((\bar{x})^{t}, \eta(t)\right)$ for $t>L$.

Let $\overline{\mathcal{S}}$ denote the spectroid formed by $(a ; 0), a \in \mathcal{S}$, and $\left((\bar{x})^{t} ; \eta(t)\right), 1<t \leq L+$ 1 , and $\mathcal{T}$ the spectroid associated with the bush $T=T(L)=\left(T_{1}^{-}, \cdots, T_{n}^{-} ; T_{1}^{+}, \cdots\right.$, $\left.T_{n}^{+} ; \sim\right)$, where the order relation on the union of the sets $T_{i}^{-}=S_{i}^{-} \amalg\left\{x_{1}, \cdots, x_{L}\right\}$, $T_{i}^{+}=S_{i}^{+} \amalg\left\{x_{1}^{\sim}, \cdots, x_{L}^{\sim}\right\}$, and $T_{l}^{\varepsilon}=S_{l}^{\varepsilon},(l, \varepsilon) \neq(i,-),(i,+)$, are defined as in case I (in particular, $x<x_{1}<\cdots<x_{L}, x_{L}^{\tilde{L}}<\cdots<x_{1}^{\sim}<x^{\sim}$ ). The equivalence relation equipped with $T$ is induced by that of $S$ and extended by $x_{j} \sim x_{j}^{\sim}$ for $j=1, \cdots, L$.

Then the correspondence

$$
(\bar{z} ; 0) \longmapsto \bar{z}, \quad z \in S, \quad\left((\bar{x})^{t} ; \eta(t)\right) \longmapsto \bar{x}_{t-1}, \quad 1<t \leq L+1
$$

defines an isomorphism from $\overline{\mathcal{S}}$ to $\mathcal{T}$.

If $\left(X ; \tilde{f_{i 2}}\right)$ contains a non-zero summand annihilated by $\mathcal{J},(X ; f)$ is isomorphic to $(x, Q)$ for some $Q \in \mathcal{P}$ because of the indecomposability of $(X ; f)$, thus is an asymmetric band.

If $\left(X ; \tilde{f_{i 2}}\right)$ does not contain a non-zero summand annihilated by $\mathcal{J}$, the reduced form $\left(\left(X ; \tilde{f_{i 2}}\right), \hat{f}\right)$ of $(X ; f)$ can be considered as a representation of the bush $T$.

By induction hypothesis, $\left(\left(X ; \tilde{f_{i 2}}\right) ; \hat{f}\right)$ is a string or a band associated with a catenation $v$ of $T$. We denote by $w$ the catenation of $S$ obtained from $v$ by replacing each term $x_{j}(j \geq 1)$ by $\underbrace{x \cdots x}_{j+1}$ and each term $x_{j}^{\sim}$ by $\underbrace{x^{\sim} \cdots x^{\sim}}_{j+1}$.

By a similar argument in case $\mathrm{I}$, there holds that $(X ; f)$ is a string or a band according as $\left(\left(X ; \tilde{f_{i 2}}\right) ; \hat{f}\right)$ is a string or a band.

4.4. Case III. $\operatorname{supp}\left(L^{-} / K^{-}\right)=\left\{\{x\},\left\{x^{\wedge}\right\}\right\}$ and $\operatorname{supp}\left(L^{+} / K^{+}\right)=\{\bar{y}\}$ for some $x, x^{\wedge} \in S_{i}^{-}$with $x \rtimes x^{\wedge}$ and some $y \in S_{i}^{+}$.

By way of example, we suppose that $y^{\sim}$ lies in $S_{j}^{-}$for some $j \neq i$. In this case, the representations $(a ; 0), a \in \mathcal{S},(\{x\} \oplus \bar{y} ; \eta(1)),\left(\left\{x^{\wedge}\right\} \oplus \bar{y} ; \eta(2)\right)$ and $\left(\{x\} \oplus\left\{x^{\wedge}\right\} \oplus \bar{y} ; \eta(3)\right)$, furnish a complete list of indecomposables in $\mathcal{B}$, where $\eta(1)=\eta(2)$ denotes the sequence $(0, \cdots, 0,1,0, \cdots, 0)$, and $\eta(3)$ the sequence 
$=(0, \cdots, 0,[11], 0, \cdots, 0)$. By $\overline{\mathcal{S}}$ we denote the spectroid formed by these representations.

Let $\mathcal{T}$ be the spectroid associated with the bush $T=\left(T_{1}^{-}, \cdots, T_{n}^{-} ; T_{1}^{+}, \cdots, T_{n}^{+}\right.$; $\sim)$, where the union of the sets $T_{i}^{-}=S_{i}^{-} \amalg\left\{x_{1}^{\prime}\right\}, T_{j}^{-}=S_{j}^{-} \amalg\left\{y_{1}^{\prime}, y_{2}^{\prime}, y_{3}^{\prime}\right\}$, and $T_{l}^{\varepsilon}=S_{l}^{\varepsilon}((l, \varepsilon) \neq(i,-),(j,-))$ is equipped with the order relation defined as in case I (in particular, $x>x_{1}^{\prime}, x^{\wedge}>x_{1}^{\prime}, y_{3}^{\prime}>y_{1}^{\prime}>y^{\sim}, y_{3}^{\prime}>y_{2}^{\prime}>y^{\sim}$ ). Finally, we equip $T$ with the equivalence relation induced by that of $S$ and extended by $x_{1}^{\prime} \sim y_{3}^{\prime}$.

Then the correspondence

$$
\begin{aligned}
(\bar{z} ; 0) \longmapsto \bar{z}, \quad z \in S, & (\{x\} \oplus \bar{y} ; \eta(1)) \longmapsto\left\{y_{1}^{\prime}\right\}, \\
\left(\left\{x^{\wedge}\right\} \oplus \bar{y} ; \eta(2)\right) \longmapsto\left\{y_{2}^{\prime}\right\}, & \left(\{x\} \oplus\left\{x^{\wedge}\right\} \oplus \bar{y} ; \eta(3)\right) \longmapsto \bar{x}_{1}^{\prime}=\bar{y}_{3}^{\prime}
\end{aligned}
$$

induces an isomorphism from $\overline{\mathcal{S}}$ and $\mathcal{T}$. Hence $\left.\left(X ; \tilde{f_{i 2}}\right) ; \hat{f}\right)$ can be viewed as a representation of the new bush $T$.

By induction hypothesis, $\left(\left(X ; \tilde{f_{i 2}}\right) ; \hat{f}\right)$ is a string or a band associated with a catenation $v$ of $T$. We denote by $w$ the catenation of $S$ obtained from $v$ by substituting $x y$ for each term $x_{1}^{\prime}, y^{\sim} x^{\wedge}$ for $y_{3}^{\prime}, y^{\sim} x y$ for $y_{1}^{\prime}$, and $y^{\sim} x^{\wedge} y$ for $y_{2}^{\prime}$.

First we suppose that $v=v_{1} v_{2} \cdots v_{s}$ is an asymmetric catenation, thus $w=$ $w_{1} w_{2} \cdots w_{t}$ is also an asymmetric catenation. We consider the following parts in $w$.

a) $w_{r} w_{r+1}=x y$ (obtained from a term $v_{q}=x_{1}^{\prime}$ in $v$ ). By construction of $R(w)$, the maps $g_{\text {wir }}$ and $f_{\text {wir }+1}$ associated with $w(2.2)$ behave as follows:

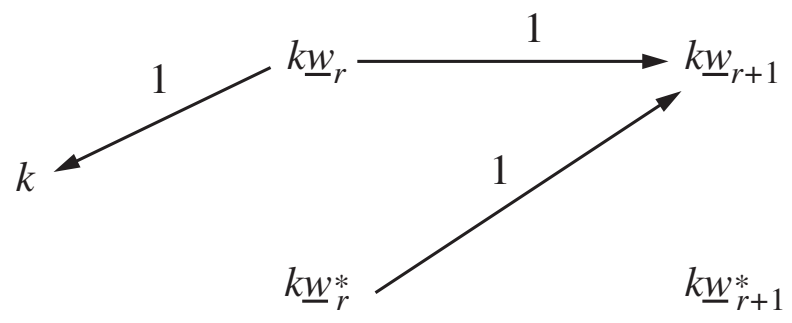

Note that $\left[w_{r-1}^{*} \ldots w_{1}^{*}\right]>\left[w_{r+1} \ldots w_{t}\right]$ since $v_{q-1}^{*}=w_{r-1}^{*}>x$ (This follows from the fact that $\left(\left(X ; \tilde{f_{i 2}}\right) ; \hat{f}\right)$ satisfies $\left.\hat{f}_{i}\left(J^{-}\left(X ; \tilde{f_{i 2}}\right)\right) \subseteq J^{+}\left(X ; \tilde{f_{i 2}}\right)\right)$ (see 3.1)). Thus every part $x y$ in $w$ provides a summand $\left(\{x\} \oplus\left\{x^{\wedge}\right\} \oplus \bar{y} ; \eta(3)\right)$ in $\left(X_{w} ; \tilde{f}_{w i 2}\right)$. Such a summand contributes a one-dimensional subspace $k\left(\underline{x}-\underline{x}^{\wedge}\right)$ in $\operatorname{Ker} f_{w i 2} \subseteq$ $N_{i}^{-}\left(X_{w} ; \tilde{f}_{w i 2}\right)$ and a one-dimensional subspace $k y^{\sim}$ in $N_{j}^{-}\left(\bar{X}_{w} ; \tilde{f}_{w i 2}\right)$.

Similarly, each part $y^{\sim} x^{\wedge}$ also provides a summand $\left.\left(\{x\} \oplus\left\{x^{\wedge}\right\} \oplus \bar{y}\right) ; \eta(3)\right)$ in $\left(X_{w} ; \tilde{f}_{w i 2}\right)$ which contributes a one-dimensional subspace both in $N_{i}^{-}\left(X_{w} ; f_{w i 2}\right)$ and in $N_{j}^{-}\left(X_{w} ; \tilde{f}_{w i 2}\right)$.

b) $w_{r} w_{r+1} w_{r+2}=y^{\sim} x y$ (obtained from $v_{q}=x_{1}^{\prime}$ ). By construction of $R(w)$, the maps $f_{w i r+1}$ and $f_{w i r+2}$ behave as follows: 


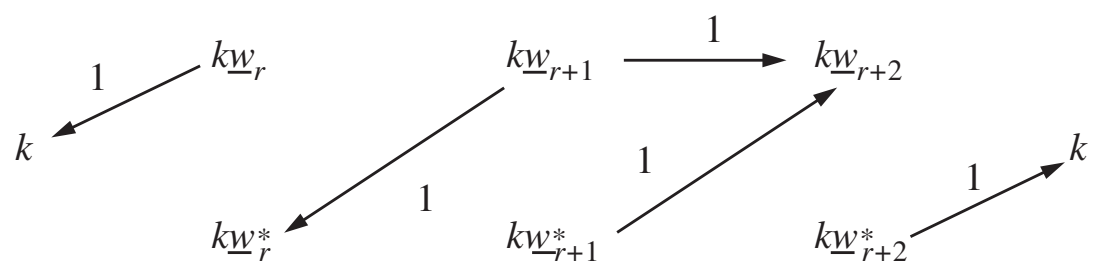

provided $\left[v_{q-1}^{*} \ldots v_{1}^{*}\right]>\left[v_{q+1} \ldots v_{s}\right]$ (thus $\left.\left[w_{r}^{*} \ldots w_{1}^{*}\right]>\left[w_{r+2} \ldots w_{t}\right]\right)$.

In this case, the part $y^{\sim} x y$ provides a summand $\left(\{x\} \oplus\left\{x^{\wedge}\right\} \oplus(\bar{y})^{2} ; \eta\right)$, where $\eta$ is of the form

$\left[\begin{array}{ll}1 & 0 \\ 1 & 1\end{array}\right]: M^{-}\left(\{x\} \oplus\left\{x^{\wedge}\right\} \oplus(\bar{y})^{2}\right)=k x \oplus k x^{\wedge} \longrightarrow k y \oplus k y=M^{+}\left(\{x\} \oplus\left\{x^{\wedge}\right\} \oplus(\bar{y})^{2}\right)$.

Such a summand contributes a two-dimensional subspace $\left(k y^{\sim}\right)^{2}$ of $N_{j}^{-}\left(X_{w} ; \tilde{f}_{w i 2}\right)$.

It is easy to see that the morphism

$$
\mu=\left[\begin{array}{cccc}
1 & 0 & 0 & 0 \\
0 & 1 & 0 & 0 \\
0 & 0 & 1 & 0 \\
0 & 0 & -1 & 1
\end{array}\right]:\{x\} \oplus\left\{x^{\wedge}\right\} \oplus(\bar{y})^{2} \longrightarrow\{x\} \oplus\left\{x^{\wedge}\right\} \oplus(\bar{y})^{2}
$$

is an isomorphism from $\left(\{x\} \oplus\left\{x^{\wedge}\right\} \oplus(\bar{y})^{2} ; \eta\right)$ to $(\{x\} \oplus \bar{y} ; \eta(1)) \oplus\left(\left\{x^{\wedge}\right\} \oplus \bar{y} ; \eta(2)\right)$.

In case $\left[v_{q-1}^{*} \ldots v_{1}^{*}\right] \leq\left[v_{q+1} \ldots v_{s}\right]$ (thus $\left[w_{r}^{*} \ldots w_{1}^{*}\right] \leq\left[w_{r+2} \ldots w_{t}\right]$ ), the maps $f_{\text {wir }+1}$ and $f_{\text {wir }+2}$ behave as follows:
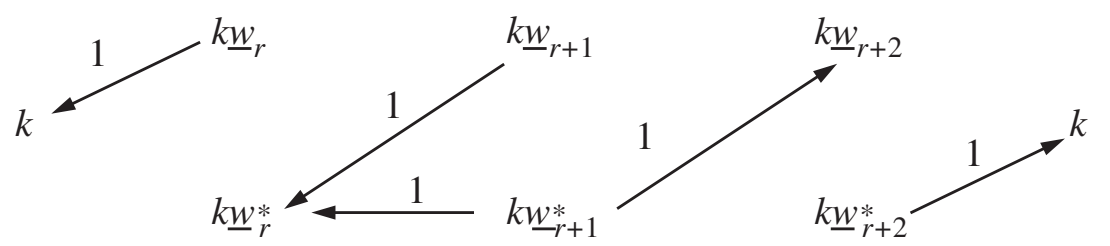

In this situation, the part $y^{\sim} x y$ provides a summand $\left.\left(\{x\} \oplus\left\{x^{\wedge}\right\} \oplus(\bar{y})^{2}\right) ; \eta^{\prime}\right)$, where $\eta^{\prime}$ is of the form

$$
\left[\begin{array}{ll}
1 & 1 \\
0 & 1
\end{array}\right]: M^{-}\left(\{x\} \oplus\left\{x^{\wedge}\right\} \oplus(\bar{y})^{2}\right)=k x \oplus k x^{\wedge} \longrightarrow k y \oplus k y=M^{+}\left(\{x\} \oplus\left\{x^{\wedge}\right\} \oplus(\bar{y})^{2}\right) .
$$

This summand also contributes a two-dimensional subspace $\left(k y^{\sim}\right)^{2}$ of $N_{j}^{-}\left(X_{w} ; \tilde{f}_{w i 2}\right)$.

It is easy to see that the morphism

$$
\mu^{\prime}=\left[\begin{array}{cccc}
1 & 0 & 0 & 0 \\
0 & 1 & 0 & 0 \\
0 & 0 & 1 & -1 \\
0 & 0 & 0 & 1
\end{array}\right]:\left(\{x\} \oplus\left\{x^{\wedge}\right\} \oplus(\bar{y})^{2}\right) \longrightarrow\left(\{x\} \oplus\left\{x^{\wedge}\right\} \oplus(\bar{y})^{2}\right)
$$


is an isomorphism from $\left(\{x\} \oplus\left\{x^{\wedge}\right\} \oplus(\bar{y})^{2} ; \eta^{\prime}\right)$ to $(\{x\} \oplus \bar{y} ; \eta(1)) \oplus\left(\left\{x^{\wedge}\right\} \oplus \bar{y} ; \eta(2)\right)$.

Therefore, every part $y^{\sim} x y$ in $w$ provides a summand in $\left(X_{w} ; \tilde{f}_{w i 2}\right)$ which is isomorphic to $(\{x\} \oplus \bar{y} ; \eta(1)) \oplus\left(\left\{x^{\wedge}\right\} \oplus \bar{y} ; \eta(2)\right)$.

Similarly, every part $y^{\sim} x^{\wedge} y$ in $w$ also provides a summand isomorphic to $(\{x\} \oplus$ $\bar{y} ; \eta(1)) \oplus\left(\left\{x^{\wedge}\right\} \oplus \bar{y} ; \eta(2)\right)$.

c) Each term $w_{r}$ (obtained from some term $v_{q}$ in $v$ ) in $w$ provides a summand $\left(\bar{w}_{r} ; 0\right)$ in $\left(X_{w} ; \tilde{f}_{w i 2}\right)$ if $w_{r} \neq w_{r}^{\sim}$, and a summand $\left.\left(\left\{w^{r}\right\} \underset{\oplus_{\tilde{f}}}{\oplus} w_{r}^{\wedge}\right\} ; 0\right)$ if $w_{r} \neq w_{r}^{\wedge}$.

Form the observations in a)-c), it follows that $\left(X_{w} ; \tilde{f}_{w i 2}\right)$ and $X_{v}$ viewed as objects in $\mathcal{B}$ are isomorphic.

Furthermore, by suitably choosing basis vectors of $N_{j}^{\varepsilon}\left(X_{w} ; \tilde{f}_{w i 2}\right)$ for $1 \leq j \leq n$ and $\varepsilon=-,+$, one can show that $\left(\left(X_{w} ; \tilde{f}_{w i 2}\right) ; \hat{f}_{w}\right)$ is isomorphic to $\left(X_{v} ; f_{v 1}, \cdots, f_{v n}\right)$. This implies that $(X ; f)$ is isomorphic to $R(w)=\left(X_{w} ; f_{w 1}, \cdots, f_{w n}\right)$, that is, $(X ; f)$ is an asymmetric string.

Similarly, one gets that $(X ; f)$ is a dimidiate string if so is $\left(\left(X ; \tilde{f_{i 2}}\right) ; \hat{f}\right)$ and that $(X ; f) \cong R(w, Q)$ (resp. $R(w, K))$ according as $\left(\left(X ; \tilde{f_{i 2}}\right) ; \hat{f}\right) \cong R(v, Q)$ (resp. $R(v, K)$ ) for some $Q \in \mathcal{P}$ (resp. $K \in \mathcal{P})$.

4.5. Case IV. $\operatorname{supp}\left(L^{-} / K^{-}\right)=\{\bar{x}\}$ and $\operatorname{supp}\left(L^{+} / K^{+}\right)=\left\{\{y\},\left\{y^{\wedge}\right\}\right\}$ for some $x \in S_{i}^{-}$and some $y, y^{\wedge} \in S_{i}^{+}$with $y \ngtr y^{\wedge}$. This is an anologue to Case III.

4.6. Case V. $\operatorname{supp}\left(L^{-} / K^{-}\right)=\left\{\{x\},\left\{x^{\wedge}\right\}\right\}$ and $\operatorname{supp}\left(L^{+} / K^{+}\right)=\left\{\{y\},\left\{y^{\wedge}\right\}\right\}$ for some $x, x^{\wedge} \in S_{i}^{-}$and $y, y^{\wedge} \in S_{i}^{+}$with $x \rtimes x^{\wedge}$ and $y \rtimes y^{\wedge}$.

In this case, one can show that the representatons $(a ; 0), a \in \mathcal{S}$, and $\mathcal{R}(E):=$ $\left((\{x\})^{s_{1}} \oplus\left(\left\{x^{\wedge}\right\}\right)^{s_{2}} \oplus(\{y\})^{t_{1}} \oplus\left(\left\{y^{\wedge}\right\}\right)^{t_{2}} ; \eta(E)\right)$, furnish a complete list of indecomposables in $\mathcal{B}$ which are not annihilated by $\mathcal{J}$, where $\eta(E)$ denotes the sequences $(0, \cdots, 0, E, 0, \cdots, 0)$ and $E$ ranges over the following matrices $(m \geq 1)$ (see Sect. 11 in $[\mathrm{GKR}])$ :

$$
\begin{aligned}
& P_{2 m-1}=\left[\begin{array}{c|c}
\mathbb{1}_{m} & 0 \\
\hline \mathbb{1}_{m-1} & 0 \\
\mathbb{1}_{m-1}
\end{array}\right] \quad\left(\begin{array}{c}
s_{2}=m-1 \\
s_{1}=t_{1}=t_{2}=m
\end{array}\right), \\
& P_{2 m-1}^{\wedge}=\left[\begin{array}{c|c}
0 & \mathbb{1}_{m} \\
\mathbb{1}_{m-1} & \\
\hline \mathbb{1}_{m-1} & \mathbb{1}_{m} \\
0 & s_{1}=m-1 \\
s_{2}=t_{1}=t_{2}=m
\end{array}\right), \\
& P_{2 m}=\left[\begin{array}{c|c}
0 & \mathbb{1}_{m} \\
\mathbb{1}_{m} & 0 \\
\hline \mathbb{1}_{m} & \mathbb{1}_{m}
\end{array}\right] \quad\left(\begin{array}{c}
s_{1}=s_{2}=t_{2}=m \\
t_{1}=m+1
\end{array}\right),
\end{aligned}
$$


Vol. $75(2000)$

$$
\begin{aligned}
& P_{2 m}^{\wedge}=\left[\begin{array}{c|c}
\mathbb{1}_{m} & \mathbb{1}_{m} \\
\hline \mathbb{1}_{m} & 0 \\
0 & \mathbb{1}_{m}
\end{array}\right] \quad\left(\begin{array}{c}
s_{1}=s_{2}=t_{1}=m \\
t_{2}=m+1
\end{array}\right), \\
& \left.I_{2 m-1}=\left[\begin{array}{c|c}
\mathbb{1}_{m} & \mathbb{1}_{m} \\
\hline \mathbb{1}_{m-1} 0 & 0 \mathbb{1}_{m-1}
\end{array}\right] \quad \begin{array}{c}
t_{2}=m-1 \\
s_{1}=s_{2}=t_{1}=m
\end{array}\right), \\
& \left.I_{2 m-1}^{\wedge}=\left[\begin{array}{c|c}
0 \mathbb{1}_{m-1} & \mathbb{1}_{m-1} 0 \\
\hline \mathbb{1}_{m} & \mathbb{1}_{m}
\end{array}\right] \quad \begin{array}{c}
t_{1}=m-1 \\
s_{1}=s_{2}=t_{2}=m
\end{array}\right), \\
& I_{2 m}=\left[\begin{array}{l|l}
0 \mathbb{1}_{m} & \mathbb{1}_{m} \\
\hline \mathbb{1}_{m} 0 & \mathbb{1}_{m}
\end{array}\right] \quad\left(\begin{array}{c}
s_{2}=t_{1}=t_{2}=m \\
s_{1}=m+1
\end{array}\right) \\
& I_{2 m}^{\wedge}=\left[\begin{array}{l|l}
\mathbb{1}_{m} & \mathbb{1}_{m} 0 \\
\hline \mathbb{1}_{m} & 0 \mathbb{1}_{m}
\end{array}\right] \quad\left(\begin{array}{c}
s_{1}=t_{1}=t_{2}=m \\
s_{2}=m+1
\end{array}\right), \\
& T_{m}^{1}=\left[\begin{array}{c|c}
\mathbb{1}_{m} & \mathbb{1}_{m} \\
+J_{m} & \\
\hline \mathbb{1}_{m} & \mathbb{1}_{m}
\end{array}\right] \quad\left(s_{1}=s_{2}=t_{1}=t_{2}=m\right) .
\end{aligned}
$$

By the finite-dimensionality of $(X ; f)$, there exists an $L>0$ such that the induced representation $\left(X ; \tilde{f_{i 2}}\right)$ does not contain a summand isomorphic to some $\mathcal{R}(E)$ for $E=P_{t}, P_{t}^{\wedge}, I_{t}, I_{t}^{\wedge}$ or $T_{t}^{1}$ with $t>L$. We then denote by $\overline{\mathcal{S}}$ the spectroid formed by $(a ; 0), a \in \mathcal{S}$, and $\mathcal{R}(E)$ for $E=P_{t}, P_{t}^{\wedge}, I_{t}, I_{t}^{\wedge}$ and $T_{t}^{1}$ wiht $1 \leq t \leq L$.

By $\mathcal{T}$ we denote the spectroid associated with the bush $T=T(L)=\left(T_{1}^{-}, \ldots\right.$, $\left.T_{n}^{-} ; T_{1}^{+}, \ldots, T_{n}^{+} ; \sim\right)$, where the sets $T_{i}^{-}=S_{i}^{-} \amalg\left\{x_{-1}, \cdots, x_{-L}, x_{1}, x_{1}^{\wedge}, \cdots, x_{L}, x_{L}^{\wedge}\right\}$, $T_{i}^{+}=S_{i}^{+} \amalg\left\{y_{-1}, \cdots y_{-L}, y_{1}, y_{1}^{\wedge}, \cdots, y_{L}, y_{L}^{\wedge}\right\}$ and $T_{l}^{\varepsilon}=S_{l}^{\varepsilon}((l, \varepsilon) \neq(i,-),(i,+))$ are equipped with order relations defined as in case I. In particular, we require that the induced order relations on $\left\{x_{-1}, \cdots, x_{-L}, x, x^{\wedge}, x_{1}, x_{1}^{\wedge}, \cdots, x_{L}, x_{L}^{\wedge}\right\}$ and $\left\{y_{-1}, \cdots, y_{-L}, y, y^{\wedge}, y_{1}, y_{1}^{\wedge}, \cdots, y_{L}, y_{L}^{\wedge}\right\}$ admit respectively the following Hassequivers: 


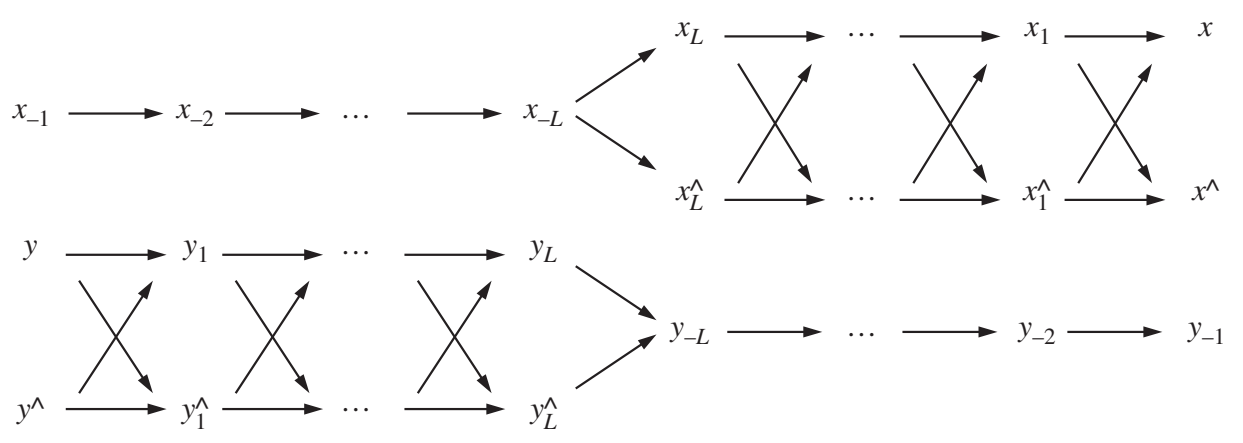

We then equip $T$ with the equivalence relation induced by $S$ and extended by $x_{-m} \sim y_{-m}, m=1, \ldots, L$.

Then the correspondence

$$
\begin{array}{rlrl}
(\bar{z} ; 0) \longmapsto \bar{z}, \quad z \in S, & \mathcal{R}\left(I_{t}\right) \longmapsto\left\{x_{t}\right\}, & \mathcal{R}\left(I_{t}^{\wedge}\right) \longmapsto\left\{x_{t}^{\wedge}\right\}, \\
1 & \leq t \leq L \\
\mathcal{R}\left(T_{t}^{1}\right) \longmapsto \bar{x}_{-t}=\bar{y}_{-t}, & \mathcal{R}\left(P_{t}\right) \longmapsto\left\{y_{t}\right\}, & \mathcal{R}\left(P_{t}^{\wedge}\right) \longmapsto\left\{y_{t}^{\wedge}\right\}
\end{array}
$$

defines an isomorphism from $\overline{\mathcal{S}}$ to $\mathcal{T}$.

If $\left(X ; \tilde{f_{i 2}}\right)$ contains a non-zero summand annihilated by $\mathcal{J},\left(\left(X ; \tilde{f_{i 2}}\right) ; \hat{f}\right)$ is isomorphic to $R(x y, K)$ for some $K \in \mathcal{Q}$, thus is a dimidiate band.

If $\left(X ; \tilde{f_{i 2}}\right)$ does not contain a non-zero summand annihilated by $\mathcal{J},\left(\left(X ; \tilde{f_{i 2}}\right) ; \hat{f}\right)$ can be considered as a representation of the bush $T$. In the following we simply identify $\bar{S}$ and $\mathcal{T}$.

By induction hypothesis, the representation $\left(\left(X ; \tilde{f_{i 2}}\right) ; \hat{f}\right)$ is a string or a band associated with a catenation $v$ of $T$. We denote by $w$ the catenation of $S$ obtained from $v$ by replacing each term $x_{m}$ by $\underbrace{x y^{\wedge} \cdots y^{\wedge} x}_{m} y \underbrace{x^{\wedge} y \cdots y x^{\wedge}}_{m}$

(resp. $\underbrace{x y^{\wedge} \cdots x y^{\wedge}}_{m} x \underbrace{y x^{\wedge} \cdots y x^{\wedge}}_{m}$ ) if $m$ is odd (resp. even),

$x_{m}^{\wedge}$ by $\underbrace{x^{\wedge} y \cdots y x^{\wedge}}_{m} y^{\wedge} \underbrace{x y^{\wedge} \cdots y^{\wedge} x}_{m}$ (resp. $\underbrace{x^{\wedge} y \cdots x^{\wedge} y}_{m} x^{\wedge} \underbrace{y^{\wedge} x \cdots y^{\wedge} x}_{m}$ ) if $m$ is odd (resp. even), $y_{m}$ by $\underbrace{y x^{\wedge} \cdots x^{\wedge} y}_{m} x \underbrace{y^{\wedge} x \cdots x y^{\wedge}}_{m}(\operatorname{resp} \cdot \underbrace{y x^{\wedge} \cdots y^{\wedge} x}_{m} y \underbrace{x y^{\wedge} \cdots x y^{\wedge}}_{m})$ if $m$ is odd (resp. even) $y_{m}^{\wedge}$ by $\underbrace{y^{\wedge} x \cdots x y^{\wedge}}_{m} x^{\wedge} \underbrace{y x^{\wedge} \cdots x^{\wedge} y}_{m}(\operatorname{resp} \cdot \underbrace{y^{\wedge} x \cdots y^{\wedge} x}_{m} y^{\wedge} \underbrace{x^{\wedge} y \cdots x^{\wedge} y}_{m})$ if $m$ is odd (resp. even), $x_{-m}^{m}$ by $\underbrace{x y^{\wedge} \cdots x y^{\wedge}}_{2 m}$, and $y_{-m}$ by $\underbrace{y x^{\sim} \cdots y x^{\sim}}_{2 m}$.

First we suppose that $v=v_{1} v_{2} \cdots v_{s}$ is finite and asymmetric, and set $w=$ $w_{1} w_{2} \cdots w_{t}$. We consider the following parts in $w$ : 
a) $w_{r} w_{r+1} \cdots w_{r+2 m-1}=x y^{\wedge} \cdots x y^{\wedge}$ (obtained from a term $x_{-m}$ in $v$ ). By construction of $R(w)$, the map $f_{w i}$ associated with $w$ acts as follows on basis vectors $\underline{w}_{r}=\underline{x}_{1}, \underline{w}_{r}^{*}=\underline{x}_{1}^{\wedge}, \cdots \cdots, \underline{w}_{r+2 m-2}=\underline{x}_{m}, \underline{w}_{r+2 m-2}^{*}=\underline{x}_{m}^{\wedge}$ :

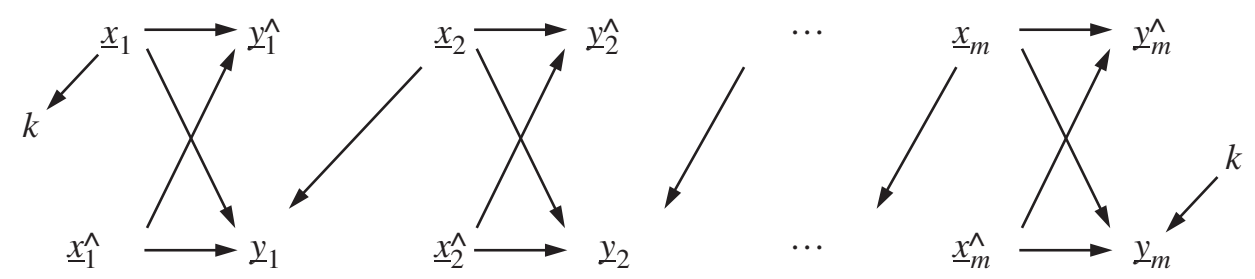

since $\left[w_{j}^{*} \ldots w_{1}^{*}\right]>\left[w_{j+2} \ldots w_{t}\right]$, for $j=r-1, \ldots, r+2 m-2$.

The matrix describing the action of $f_{w i}$ on the basis vectors $\underline{x}_{i}, \underline{x}_{i}^{\wedge}, 1 \leq i \leq m$ in the figure above is

$$
\left[\begin{array}{lllll|lllll}
1 & 1 & \cdot & 0 & 0 & 1 & 0 & \cdot & 0 & 0 \\
0 & 1 & \cdot & 0 & 0 & 0 & 1 & \cdot & 0 & 0 \\
\cdot & \cdot & \cdot & \cdot & \cdot & \cdot & \cdot & \cdot & \cdot & \cdot \\
0 & 0 & \cdot & 1 & 1 & 0 & 0 & \cdot & 1 & 0 \\
0 & 0 & \cdot & 0 & 1 & 0 & 0 & \cdot & 0 & 1 \\
\hline 1 & 0 & \cdot & 0 & 0 & 1 & 0 & \cdot & 0 & 0 \\
0 & 1 & \cdot & 0 & 0 & 0 & 1 & \cdot & 0 & 0 \\
\cdot & \cdot & \cdot & \cdot & \cdot & \cdot & \cdot & \cdot & \cdot & \cdot \\
0 & 0 & \cdot & 1 & 0 & 0 & 0 & \cdot & 1 & 0 \\
0 & 0 & \cdot & 0 & 1 & 0 & 0 & \cdot & 0 & 1
\end{array}\right]=T_{m}^{1}
$$

Thus every part $\underbrace{x y^{\wedge} \cdots x y^{\wedge}}_{2 m}$ in $w$ provides a summand in $\left(X_{w} ; \tilde{f}_{w i 2}\right)$ which is isomorphic to $\mathcal{R}\left(T_{m}^{1}\right)$, and such a summand contributes a one-dimensional subspace both in $N_{i}^{-}\left(X_{w} ; \tilde{f}_{w i 2}\right)$ and in $N_{i}^{+}\left(X_{w} ; \tilde{f}_{w i 2}\right)$.

Similarly, every part $\underbrace{y^{\wedge} x \cdots y^{\wedge} x}_{2 m}$ (obtained from some term $y_{-m}$ in $v$ ) provides a summand isomorphic to $\mathcal{R}\left(T_{m}^{1}\right)$, too.

b) $w_{r} w_{r+1} \cdots w_{r+2 m}=x y^{\wedge} \cdots y x^{\wedge}$ (obtained from a term $v_{q}=x_{m}$ ).

Case 1. $m=2 p-1$. By construction of $R(w)$, the map $f_{w i}$ associated with $w$ acts as follows on basis vectors $\underline{w}_{r}=\underline{x}_{1}, \underline{w}_{r}^{*}=\underline{x}_{1}^{\wedge}, \cdots \cdots, \underline{w}_{r+2 m}=\underline{x}_{2 p}^{\wedge}, \underline{w}_{r+2 m}^{*}=$ $\underline{x}_{2 p}$ :

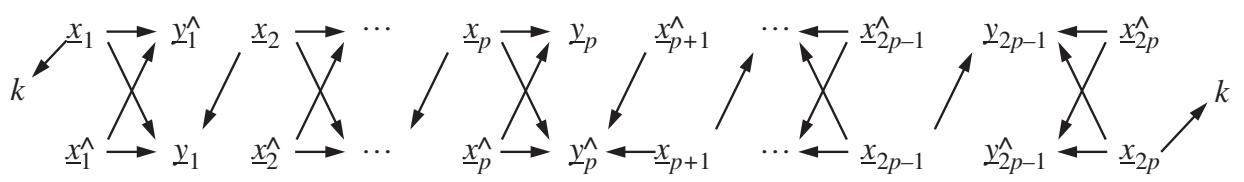


provided $\left[v_{q-1}^{*} \cdots v_{1}^{*}\right]>\left[v_{q+1} \cdots v_{s}\right]\left(\right.$ Hence $\left.\left[w_{r+2 p-1}^{*} \cdots w_{1}^{*}\right]>\left[w_{r+2 p+1} \cdots w_{t}\right]\right)$.

We start with the following change of basis vectors.

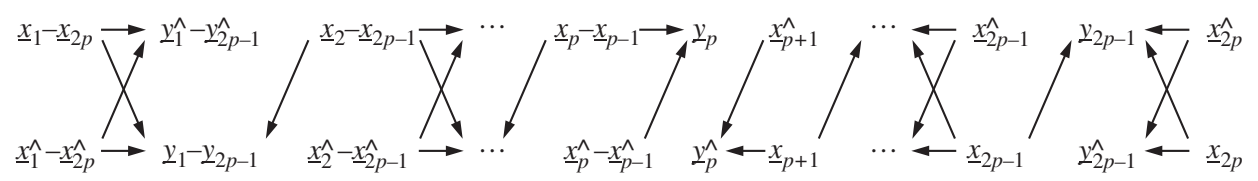

Thus, the part $w_{r} w_{r+1} \cdots w_{r+2 m}=\underbrace{x y^{\wedge} \cdots y^{\wedge} x}_{m} y \underbrace{x^{\wedge} y \cdots y x^{\wedge}}_{m}$ porvides a summand of $\left(X_{w} ; \tilde{f}_{w i 2}\right)$ which is isomorphic to $\mathcal{R}(Y) \oplus \mathcal{R}\left(Y^{\wedge}\right)$, where $Y$ and $Y^{\wedge}$ are the following matrices:

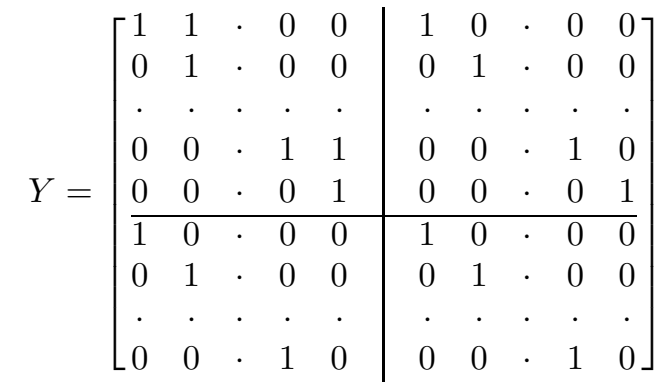

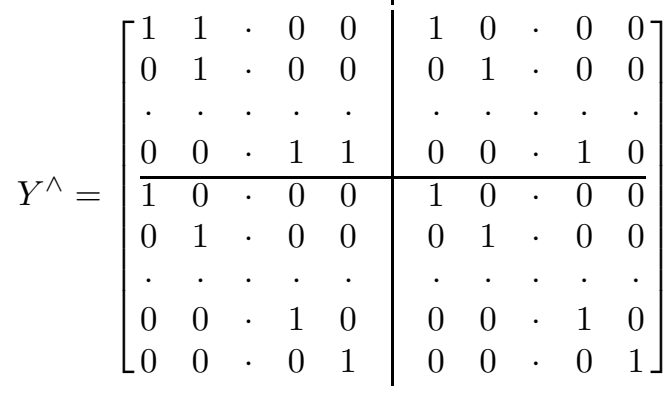

Set

$$
U_{p}=\left[\begin{array}{cc}
0 & \exp R_{p-1} \\
H_{p}
\end{array}\right] \in k^{p \times p}
$$

where

$$
R_{p}=\left[\begin{array}{ccccc}
0 & 0 & \cdot & 0 & 0 \\
-1 & 0 & \cdot & 0 & 0 \\
0 & -2 & \cdot & 0 & 0 \\
\cdot & \cdot & \cdot & \cdot & \cdot \\
0 & 0 & \cdot & -p+1 & 0
\end{array}\right] \in k^{p \times p}
$$


and $H_{p}$ denotes the last row of the matrix $\exp R_{p}$. Then there holds that

$$
\left[\begin{array}{c|c}
U_{p} & 0 \\
\hline 0 & \exp R_{p-1}
\end{array}\right] I_{2 p-1}=Y\left[\begin{array}{c|c}
\exp R_{p} & 0 \\
\hline 0 & U_{p}
\end{array}\right]
$$

and

$$
\left[\begin{array}{c|c}
\exp R_{p-1} & 0 \\
\hline 0 & \exp R_{p}
\end{array}\right] I_{2 p-1}^{\wedge}=Y^{\wedge}\left[\begin{array}{c|c}
\exp R_{p} & 0 \\
\hline 0 & \exp R_{p}
\end{array}\right]
$$

that is, the morphism

$$
\begin{array}{r}
\exp R_{p} \oplus U_{p} \oplus U_{p} \oplus \exp R_{p-1}:\{x\}^{p} \oplus\left\{x^{\wedge}\right\}^{p} \oplus\{y\}^{p} \oplus\left\{y^{\wedge}\right\}^{p-1} \\
\longrightarrow\{x\}^{p} \oplus\left\{x^{\wedge}\right\}^{p} \oplus\{y\}^{p} \oplus\left\{y^{\wedge}\right\}^{p-1}
\end{array}
$$

defines an ismorphism from $\mathcal{R}(Y)$ to $\mathcal{R}\left(I_{2 p-1}\right)$, and the morphism

$$
\begin{array}{r}
\exp R_{p} \oplus \exp R_{p} \oplus \exp R_{p-1} \oplus \exp R_{p}:\{x\}^{p} \oplus\left\{x^{\wedge}\right\}^{p} \oplus\{y\}^{p-1} \oplus\left\{y^{\wedge}\right\}^{p} \\
\longrightarrow\{x\}^{p} \oplus\left\{x^{\wedge}\right\}^{p} \oplus\{y\}^{p-1} \oplus\left\{y^{\wedge}\right\}^{p}
\end{array}
$$

defines an ismorphism from $\mathcal{R}\left(Y^{\wedge}\right)$ to $\mathcal{R}\left(I_{2 p-1}^{\wedge}\right)$.

Therefore, every part $\underbrace{x y^{\wedge} \cdots y^{\wedge} x}_{m} y \underbrace{x^{\wedge} y \cdots y x^{\wedge}}_{m}$ in $w$ porvides a summand of $\left(X ; \tilde{f_{i 2}}\right)$ which is isomorphic to $\mathcal{R}\left(I_{2 p-1}\right) \oplus \mathcal{R}\left(I_{2 p-1}^{\wedge}\right)$ and which contributes a twodimensional subspace in $N_{i}^{-}\left(X_{w} ; \tilde{f}_{w i 2}\right)$.

The case $\left[v_{q-1}^{*} \cdots v_{1}^{*}\right] \leq\left[v_{q+1} \cdots v_{s}\right]$ can be treated similarly.

Case 2. $m=2 p$. By construction of $R(w)$, the map $f_{w i}$ acts as follows on basis vectors $\underline{w}_{r}=\underline{x}_{1}, \underline{w}_{r}^{*}=\underline{x}_{1}^{\wedge}, \cdots \cdots, \underline{w}_{r+2 m}=\underline{x}_{2 p+1}^{\wedge}, \underline{w}_{r+2 m}^{*}=\underline{x}_{2 p+1}$ :

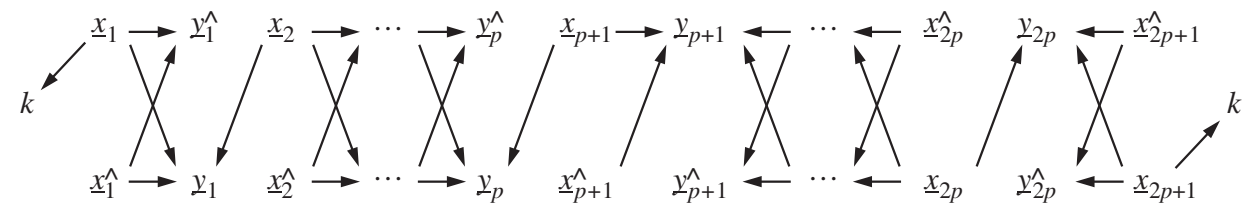

provided $\left[v_{q-1}^{*} \cdots v_{1}^{*}\right]>\left[v_{q+1} \cdots v_{s}\right]$.

We start with the following change of basis vectors.

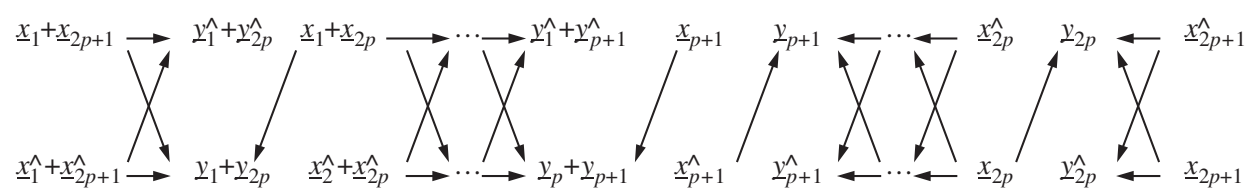


Thus, in this case, the part $w_{r} w_{r+1} \cdots w_{r+2 m}=\underbrace{x y^{\wedge} \cdots x y^{\wedge}}_{m} x \underbrace{y x^{\wedge} \cdots y x^{\wedge}}_{m}$ provides a summand of $\left(X_{w} ; \tilde{f}_{w i 2}\right)$ which is isomorphic to $\mathcal{R}(Z) \oplus \mathcal{R}\left(Z^{\wedge}\right)$, where $Z$ and $Z^{\wedge}$ are the following matrices:

$$
\begin{aligned}
& Z=\left[\begin{array}{llllll|lllll}
1 & 1 & \cdot & 0 & 0 & 0 & 1 & 0 & \cdot & 0 & 0 \\
0 & 1 & \cdot & 0 & 0 & 0 & 0 & 1 & \cdot & 0 & 0 \\
\cdot & \cdot & \cdot & \cdot & \cdot & \cdot & \cdot & \cdot & \cdot & \cdot & \cdot \\
0 & 0 & \cdot & 1 & 1 & 0 & 0 & 0 & \cdot & 1 & 0 \\
0 & 0 & \cdot & 0 & 1 & 1 & 0 & 0 & \cdot & 0 & 1 \\
\hline 1 & 0 & \cdot & 0 & 0 & 0 & 1 & 0 & \cdot & 0 & 0 \\
0 & 1 & \cdot & 0 & 0 & 0 & 0 & 1 & \cdot & 0 & 0 \\
\cdot & \cdot & \cdot & \cdot & \cdot & \cdot & \cdot & \cdot & \cdot & \cdot & \cdot \\
0 & 0 & \cdot & 1 & 0 & 0 & 0 & 0 & \cdot & 1 & 0 \\
0 & 0 & \cdot & 0 & 1 & 0 & 0 & 0 & \cdot & 0 & 1
\end{array}\right]
\end{aligned}
$$

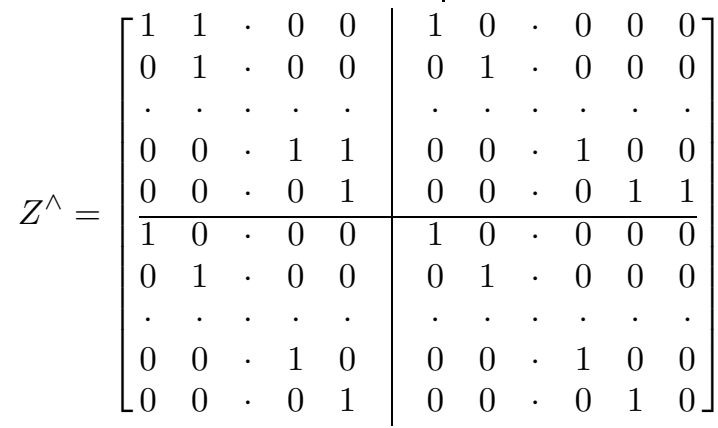

then there hold that

$$
\begin{gathered}
{\left[\begin{array}{c|c}
\exp R_{p} & 0 \\
\hline 0 & \exp R_{p}
\end{array}\right] I_{2 p}=Z\left[\begin{array}{c|c}
\exp R_{p+1} & 0 \\
\hline 0 & \exp R_{p}
\end{array}\right]} \\
{\left[\begin{array}{c|c}
U_{p} & 0 \\
\hline 0 & \exp R_{p}
\end{array}\right] I_{2 p}^{\wedge}=Z^{\wedge}\left[\begin{array}{c|c}
\exp R_{p} & 0 \\
\hline 0 & V_{p+1}
\end{array}\right]}
\end{gathered}
$$

where $U_{p}$ and $R_{p}$ are defined as before and $V_{p+1}$ has the form

$$
V_{p+1}=\left[\begin{array}{cc}
0 & \exp R_{p} \\
& -H_{p+1}
\end{array}\right] \in k^{(p+1) \times(p+1)}
$$

This implies that the morphism

$$
\begin{array}{r}
\exp R_{p+1} \oplus \exp R_{p} \oplus \exp R_{p} \oplus \exp R_{p}:\{x\}^{p+1} \oplus\left\{x^{\wedge}\right\}^{p} \oplus\{y\}^{p} \oplus\left\{y^{\wedge}\right\}^{p} \\
\longrightarrow\{x\}^{p+1} \oplus\left\{x^{\wedge}\right\}^{p} \oplus\{y\}^{p} \oplus\left\{y^{\wedge}\right\}^{p}
\end{array}
$$


defines an ismorphism from $\mathcal{R}(Z)$ to $\mathcal{R}\left(I_{2 p}\right)$, and the morphism

$$
\begin{array}{r}
\exp R_{p} \oplus V_{p+1} \oplus U_{p} \oplus \exp R_{p}:\{x\}^{p} \oplus\left\{x^{\wedge}\right\}^{p+1} \oplus\{y\}^{p} \oplus\left\{y^{\wedge}\right\}^{p} \\
\longrightarrow\{x\}^{p} \oplus\left\{x^{\wedge}\right\}^{p+1} \oplus\{y\}^{p} \oplus\left\{y^{\wedge}\right\}^{p}
\end{array}
$$

defines an ismorphism from $\mathcal{R}\left(Z^{\wedge}\right)$ to $\mathcal{R}\left(I_{2 p}^{\wedge}\right)$.

Hence each part $\underbrace{x y^{\wedge} \cdots x y^{\wedge}}_{m} x \underbrace{y x^{\wedge} \cdots y x^{\wedge}}_{m}$ in $w$ porvides a summand of $\left(X_{w} ; \tilde{f}_{w i 2}\right)$ which is isomorphic to $\mathcal{R}\left(I_{2 p}\right) \oplus \mathcal{R}\left(I_{2 p}^{\wedge}\right)$ and which contributes a two-dimensional subspace in $N_{i}^{-}\left(X_{w} ; \tilde{f}_{w i 2}\right)$.

The case $\left[v_{q-1}^{*} \cdots v_{1}^{*}\right] \leq\left[v_{q+1} \cdots v_{s}\right]$ is similar.

$\left.\mathrm{b}^{\prime}\right) w_{r} w_{r+1} \cdots w_{r+2 m}=x^{\wedge} y \cdots y^{\wedge} x$ (obtained from a term $v_{q}=x_{m}^{\wedge}$ ). As in b), one has that every part $\underbrace{x^{\wedge} y \cdots y^{\wedge} x}_{2 m+1}$ provides a summand in $\left(X_{w} ; \tilde{f}_{w i 2}\right)$ which is isomorphic to $\mathcal{R}\left(I_{m}\right) \oplus \mathcal{R}\left(I_{m}^{\wedge}\right)$.

c) By a similar argument in b), one can show that both the parts $\underbrace{y x^{\wedge} \cdots x y^{\wedge}}_{2 m+1}$ (obtained from a term $v_{q}=y_{m}$ ) and $\underbrace{y^{\wedge} x \cdots x^{\wedge} y}_{2 m+1}$ (obtained from a term $v_{q}^{\wedge}=y_{m}$ ) provides a summand in $\left(X_{w} ; \tilde{f}_{w i 2}\right)$ which is isomorphic to $\mathcal{R}\left(P_{m}\right) \oplus \mathcal{R}\left(P_{m}^{\wedge}\right)$.

d) Each term $w_{r}$ (obtained from some term $v_{q}$ in $v$ ) in $w$ provides a summand $\left(\bar{w}_{r} ; 0\right)$ in $\left(X_{w} ; \tilde{f}_{w i 2}\right)$ if $w_{r} \neq w_{r}^{\sim}$, and a summand $\left(\left\{w^{r}\right\} \oplus\left\{w_{r}^{\wedge}\right\} ; 0\right)$ if $w_{r} \neq w_{r}^{\wedge}$.

From the observations in a)-d), one gets that $\left(X_{w} ; \tilde{f}_{w i 2}\right)$ and $X_{v}$ viewed as objects in $\mathcal{B}$ are isomorphic.

Furthermore, by checking each summand described in a)-d) and suitably choosing basis vectors of $N_{j}^{\varepsilon}\left(X_{w} ; \tilde{f}_{w i 2}\right)$ for $1 \leq j \leq n$ and $\varepsilon=-,+$, one obtains that $\left(\left(X_{w} ; \tilde{f}_{w i 2}\right) ; \hat{f}_{w}\right)$ is isomorphic to $\left(X_{v} ; f_{v 1}, \cdots, f_{v n}\right)$. Thus $(X ; f)$ is isomorphic to $R(w)$, that is, an asymmetric string.

Similarly, one can show the following:

(1) If $v$ is a symmetric catenation, there holds that $(X ; f)$ is isomorphic to a non-trivial summand of $\left(X_{w} ; f_{w 1}, \cdots, f_{w n}\right)$, that is, a dimidiate string.

(2) If $v$ is an asymmetric periodic catenation of period $\pi$ and $\left(\left(X ; \tilde{f_{i 2}}\right) ; \hat{f}\right) \cong$ $R(v, Q)$ for some $Q \in \mathcal{P}$, there holds that $(X ; f) \cong R(w, \bar{Q})$, where $\bar{Q}$ denotes the polynomial $\bar{Q}(X)=(-1)^{\iota} \operatorname{deg} Q Q\left((-1)^{\iota} X\right)$, and $\iota$ is the number of terms $x_{m}, x_{m}^{\wedge}$, $y_{m}$, and $x_{m}^{\wedge}$ in $v=v_{0} v_{1} \cdots v_{\pi-1}$ with $m$ an odd number.

(3) If $v$ is symmetric periodic and $\left(\left(X ; \tilde{f_{i 2}}\right) ; \hat{f}\right) \cong R(v, K)$ for some $K \in \mathcal{Q}$, there holds that $(X ; f) \cong R(u, K)$.

4.7. As a conclusion of $4.2-4.6$, we have the following

Proposition. Each indecomposable representation of the bush $S$ is a string or a band. 
4.8. Throughout the reduction above, by substituting $\left(X ; f_{1}, \cdots, f_{n}\right)$ for representations associated with catenations, one can prove inductively the following propositions.

Proposition 1. (1) The representation $R(w)$ associated with each asymmetric catenation $w$ is indecomposable, and the representation $\left(X_{v} ; f_{v 1}, \cdots, f_{v n}\right)$ associated with each symmetric catenation $v$ is a direct sum of two non-isomorphic indecomposables.

(2) For each asymmetric (resp. a symmetric) periodic catenation and each $Q \in$ $\mathcal{P}($ resp. $K \in \mathcal{Q})$, the representation $R(u, Q)$ (resp. $R(u, K))$ is indecomposable.

(3) The representaions $R(\delta)$, where

$$
\delta \in \Omega_{1} \amalg \Omega_{2} \times\{0,1\} \amalg \Omega_{3} \times \mathcal{P} \amalg \Omega_{4} \times \mathcal{Q},
$$

are pairwise non-isomorphic.

Proposition 2. The equivalent catenations (finite or periodic) of $S$ define the same family of isoclasses of indecomposables.

Proof. Let $w=w_{1} w_{2} \cdots w_{t}$ and $w^{\prime}=w_{1}^{\prime} w_{2}^{\prime} \cdots w_{t}^{\prime}$ be equivalent catenations. We denote by $d\left(w, w^{\prime}\right)$ the number of indices $i(1 \leq i \leq t)$ such that $w_{i}^{\prime} \neq w_{i}$. If $d\left(w, w^{\prime}\right)=0$, the proposition holds. If $d\left(w, w^{\prime}\right)>1$, there exists a sequence of equivalent catenations $W_{1}=w, W_{2}, \cdots, W_{d}=w^{\prime}$ such that $d\left(W_{i}, W_{i+1}\right)=1$ for $1 \leq i \leq d-1$. So we may suppose that $d\left(w, w^{\prime}\right)=1$. Applying the reduction in $4.2-4.6$, the representations $\left(X_{w} ; f_{w 1}, \cdots, f_{w n}\right)$ and $\left(X_{w^{\prime}} ; f_{w^{\prime} 1}, \cdots, f_{w^{\prime} n}\right)$ are respectively reduced to representations $\left(X_{v} ; f_{v 1}, \cdots, f_{v n}\right)$ and $\left(X_{v^{\prime}} ; f_{v^{\prime}}, \cdots, f_{v^{\prime} n}\right)$ of a new bush $T$, where $v$ and $v^{\prime}$ are equivalent catenations of $T$ such that $d\left(v, v^{\prime}\right) \leq$ 1. By induction, we may suppose that $v$ and $v^{\prime}$ define the same family of isoclasses of indecomposables of T. By Proposition 3.4, this implies that $w$ and $w^{\prime}$ defines the same family of isoclasses of indecomposables of $S$.

By a similar argument, the proposition holds for periodic catenations.

4.9. Remark. By Proposition 2 in 4.8 and Remark 2.8, one obtains the following statements.

(a) For each asymmetric catenation $w$, catenations in the class $[w] \amalg\left[w^{*}\right]$ define isomorphic representations.

(b) For each symmetric catenation $w$, catenations in the class $[w]$ define isomorphic representations.

(c) For each asymmetric periodic catenation $u$, catenations in the class

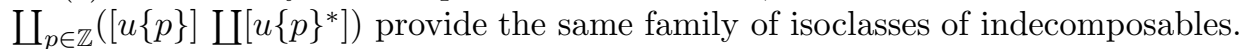

(d) For each symmetric catenation $w$, catenations in the class $\coprod_{p \in \mathbb{Z}}[u\{p\}]$ provide the same family of isoclasses of indecomposables.

The main theorem then follows from Propositions in 4.7, 4.8, and Remark 4.9. 


\section{Acknowledgements}

The present paper is a revised version of my dissertation. The author is grateful to Professor P. Gabriel for his encouragement and his many valuable suggestions in the preparation of this paper. We would like to thank Professor Sergejchuk for pointing out to me the work of Bondarenko.

\section{References}

[1] V. M.Bondarenko, Representations of bundles of semichained sets and their applications, St. Peterburg Math. J. 5 (3) (1992), 973-996.

[2] W. W. Crawley-Boevey, Functorial filtrations II: clans and the Gelfand problem, J. London Math. Soc. 40 (2) (1989), 9-30.

[3] B. Deng, An algorithm and self-reproducing systems, Comm. Algebra 26 (1998), 34193434.

[4] P. Gabriel, A historical recording. Finite-dimensional representations of the algebra $\mathcal{A}=$ $k[[a, b]] /(a b)$ after Gelfand-Ponomarev, A literal translation of notes in German reproducing a lecture at a seminar organised in Bonn during the winter term 1973/74. 16 pages, April 1991.

[5] P. Gabriel, B. Keller, L. A. Roiter, Representations of finite-dimensional algebras, Encycl. Math. Sc. 73 (1992), 1-176.

[6] I. M. Gelfand, The cohomology of infinite dimensional Lie algebras, some questions of integral geometry, Actes du Congres International des Mathematician, Nice 1970.

[7] I. M. Gelfand, V. A. Ponomarev, Indecomposable representations of Lorentz group, Russian Math. Survey 23 (1968), 1-58.

[8] L. A. Nazarova, A. V. Roiter, A problem of I.M. Gelfand, Funktsional'nyi Analiz i Ego Prilozheniya 7 (1973), 54-69.

[9] C. M. Ringel, The indecomposable representations of the dihedral 2-groups, Math. Ann. 214 (1975), 19-34.

Bangming Deng

Department of Mathematics

Beijing Normal University

100875 Beijing

P.R.China

e-mail: dengbm@bnu.edu.cn

(Received: April 10, 1997 ) 